\title{
Non-polynomial cubic spline discretization for system of non-linear singular boundary value problems using variable mesh
}

Ranjan Kumar Mohanty ${ }^{1 *}$, Sucheta Nayak ${ }^{2,3}$ and Arshad Khan ${ }^{3}$

*Correspondence:

rmohanty@sau.ac.in

${ }^{1}$ Department of Mathematics,

South Asian University, New Delhi,

110021, India

Full list of author information is

available at the end of the article

\begin{abstract}
In this paper, we propose two generalized non-polynomial cubic spline schemes using a variable mesh to solve the system of non-linear singular two point boundary value problems. Theoretical analysis proves that the proposed methods have secondand third-order convergence. Both methods are applicable to singular boundary value problems. Numerical results are also provided to show the accuracy and efficiency of the proposed methods.
\end{abstract}

Keywords: non-polynomial; cubic spline; variable mesh; singular; non-linear

\section{Introduction}

In this paper, we study two effective numerical techniques using a non-polynomial cubic spline based on a variable mesh to solve system of $M$ non-linear singular boundary value problems (BVPs) of the following type:

$$
y_{x x}^{(i)}=F^{(i)}\left(x, y^{(1)}, \ldots, y^{(i)}, \ldots, y^{(M)}, y_{x}^{(1)}, \ldots, y_{x}^{(i)}, \ldots, y_{x}^{(M)}\right), \quad a \leq x \leq b,
$$

subject to boundary conditions

$$
y^{(i)}(a)=A_{i}, \quad y^{(i)}(b)=B_{i}, \quad \text { where } y_{x}^{(i)}=\frac{d y^{(i)}}{d x}, y_{x x}^{(i)}=\frac{d^{2} y^{(i)}}{d x^{2}}, i=1,2,3, \ldots, M
$$

We assume that, for $-\infty<a \leq x \leq b<\infty$ and $-\infty<y^{(i)}, y_{x}^{(i)}<\infty$, where $y^{(i)}=y^{(i)}(x), y_{x}^{(i)}=$ $y_{x}^{(i)}(x)$, we have

(i) $F^{(i)}\left(x, y^{(1)}, y^{(2)}, \ldots, y^{(i)}, \ldots, y^{(M)}, y_{x}^{(1)}, y_{x}^{(2)}, \ldots, y_{x}^{(i)}, \ldots, y_{x}^{(M)}\right)$ is continuous;

(ii) $\frac{\partial F^{(i)}}{\partial y^{(j)}}$ and $\frac{\partial F^{(i)}}{\partial y_{x}^{(j)}}$ exist and are continuous;

(iii) $\frac{\partial F^{(i)}}{\partial y^{(j)}}>0$ and $\left|\frac{\partial F^{(i)}}{\partial y_{x}^{(j)}}\right| \leq C$, for some positive constant $C$ and $i, j=1,2,3, \ldots, M$.

These conditions as proved by Keller [1] ensure us of the existence of a unique solution of the above system of boundary value problem (1)-(2).

(c) The Author(s) 2017. This article is distributed under the terms of the Creative Commons Attribution 4.0 International License (http://creativecommons.org/licenses/by/4.0/), which permits unrestricted use, distribution, and reproduction in any medium, provided you give appropriate credit to the original author(s) and the source, provide a link to the Creative Commons license, and indicate if changes were made. 
In the present paper, we have derived generalized non-polynomial cubic spline schemes of second- and third-order using a variable mesh for solving system of two point boundary value problems (1)-(2). Such systems effectively decompose several higher-order problems into second-order boundary value problems; thus solving them efficiently. These higherorder problems are used to model various phenomena in the field of astrophysics, astronomy, hydrodynamics, beam and wave theory [2-6]. For these boundary value problems, Aftabizadeh [7], Zill [8], Regan [9] and Agarwal [10] have obtained the existence and uniqueness of the solutions.

Many authors have developed efficient numerical schemes to solve boundary value problems and splines have been rigourously used to approximate the solution of such problems. To name a few, Mohanty et al. [11-13] developed AGE, cubic spline TAGE, Newton-TAGE iteration methods using a finite difference and cubic spline method based on uniform and non-uniform mesh, respectively, to solve non-linear singular two point boundary value problems. Even singularly perturbed boundary value problems with or without first derivative terms are solved. Kadalbajoo et al. [14] developed a third-order variable-mesh cubic spline method; Mohanty et al. used a spline in compression $[15,16]$, a spline in tension [17, 18], and the cubic spline TAGE method [19]. Wazwaz [20] developed modified Adomian decomposition method to solve linear and non-linear fourthorder boundary value problems, Akram and Siddiqi [21] used the second-order convergent non-polynomial spline method to solve sixth-order linear special case boundary value problems. Talwar and Mohanty [22] developed a finite-difference method for the solution of a fourth-order ordinary differential equation. Twizell and Boutayeb [23] developed finite-difference methods for solving eighth-order boundary value problems. Akram and Rehman [24] solved eighth-order boundary value problems using the kernel space method. Siddiqi and Akram [25, 26] solved sixth- and eighth-order boundary value problems using a non-polynomial and septic spline. Liu and $\mathrm{Wu}$ [27] used a generalized differential quadrature rule to solve a special case of eighth-order boundary value problems. Also a variable mesh has been extensively used by many authors. Numerical simulations with high-order compact difference schemes depict more accurate solution values on variable meshes as compared to some high-order compact scheme on a uniform mesh network. This happens because the truncation error in a finite-difference approximation depends upon the derivative of the variable as well as mesh spacing. Therefore, to attain uniformly distributed truncation errors, it is essential to employ non-uniform meshes, i.e., finer meshes in the region for largely deviated derivatives and coarse meshes for a smooth function. In this manner, the error disperses almost uniformly over the domain of integration and renders an accurate solution to a greater extent [28]. Such ongoing work motivated us to develop an efficient non-polynomial cubic spline scheme to solve the system of non-linear singular boundary value problems using a variable mesh.

Presently, once the proposed method decomposes the higher-order boundary value problem into a system of second-order boundary value problems (1), the boundary conditions are also accordingly modified. As the boundary conditions are incorporated, we get a block tri-diagonal Jacobian from the system of discretized second-order boundary value problems. We implemented the method on non-linear as well as linear problems. In the case of a linear boundary value problem, we get a linear system of coupled difference equations, which are solved by a block Gauss elimination method and the non- 
linear system of coupled difference equations are solved by using the block Newton's method.

The sections of this paper are organized as follows. In Section 2, we give details of derivation of the scheme using a second-order singular linear boundary value problem and, in Section 3, we provide a generalization of the scheme. In Section 4, we present the application of the proposed schemes to a fourth- and sixth-order singular BVP. In Section 5, we discuss a convergence analysis and in Section 6, we provide numerical illustrations to demonstrate the accuracy of the proposed schemes. Finally in Section 7, we provide concluding remarks.

\section{Derivation of the schemes}

We consider a scalar second-order non-linear boundary value problem of the following type:

$$
y_{x x}=F\left(x, y, y_{x}\right), \quad \text { such that } y(a)=A, y(b)=B .
$$

Firstly, we discretize the solution region $[a, b]$ such that $a=x_{0}<x_{1}<x_{2}<\cdots<x_{N-1}<$ $x_{N}=b$. Let $h_{j}=x_{j}-x_{j-1}, j=1,2,3, \ldots, N$, be the mesh size and the mesh ratio be $\sigma=\frac{h_{j+1}}{h_{j}}>0$, $j=1,2,3, \ldots, N-1$. When $\sigma=1$, the mesh reduces to a uniform mesh, i.e., $h_{j+1}=h_{j}=h$. Also, assume $y_{j}$ and $Y_{j}$ to be the approximate and exact solution of (3) at the grid points $x_{j}$, $j=1,2, \ldots, N$. Then the interpolating non-polynomial cubic spline approximation function can be defined as

$$
\begin{aligned}
S(x) & =a_{j}+b_{j}\left(x-x_{j}\right)+c_{j} \sin \left(k\left(x-x_{j}\right)\right)+d_{j} \cos \left(k\left(x-x_{j}\right)\right), \\
k & >0, x_{j-1} \leq x \leq x_{j}, j=1,2,3, \ldots, N,
\end{aligned}
$$

which satisfies the following conditions:

(i) $S(x)$ coincides with a cubic polynomial in $\left[x_{j-1}, x_{j}\right], j=1,2,3, \ldots, N$.

(ii) $S(x) \in C^{2}[a, b]$.

(iii) $S\left(x_{j}\right)=y\left(x_{j}\right), S_{x x_{j}}\left(x_{j}\right)=F_{j}, S_{x x_{j}}\left(x_{j \pm \frac{1}{2}}\right)=F_{j \pm \frac{1}{2}}$ for $j=1,2,3, \ldots, N$.

Using the definition of spline (4) and conditions (i) and (iii) we get the following values for $a_{j}, b_{j}, c_{j}, d_{j}$ and approximations:

$$
\begin{aligned}
& a_{j}=y_{j}+\frac{F_{j}}{k^{2}}, \quad b_{j}=\frac{y_{j+1}-y_{j}}{h_{j}}+\frac{F_{j+1}-F_{j}}{k^{2} h_{j}}, \\
& c_{j}=-\frac{F_{j}}{k^{2}} \quad \text { and } \quad d_{j}=\frac{F_{j} \cos \left(k h_{j}\right)-F_{j+1}}{k^{2} \sin \left(k h_{j}\right)}, \\
& S_{x}\left(x_{j+1}\right)=\frac{y_{j+1}-y_{j}}{h_{j+1}}+h_{j+1}\left(\alpha F_{j}+\beta_{1} F_{j+1}\right), \\
& S_{x}\left(x_{j-1}\right)=\frac{y_{j}-y_{j-1}}{h_{j}}-h_{j}\left(\gamma F_{j}+\beta_{2} F_{j-1}\right), \\
& S_{x}\left(x_{j}\right)=\frac{y_{j+1} \beta_{3}+y_{j}\left(\sigma_{j}^{2} \beta_{4}-\beta_{3}\right)-y_{j-1} \sigma_{j}^{2} \beta_{4}}{h_{j} \sigma_{j}}-h_{j+1}\left(\alpha \beta_{3} F_{j+1}-\gamma \beta_{4} F_{j-1}\right),
\end{aligned}
$$


where

$$
\begin{aligned}
& \alpha=\frac{-1}{\theta_{j+1}^{2}}\left(1-\frac{\theta_{j+1}}{\sin \theta_{j+1}}\right), \quad \gamma=\frac{-1}{\theta_{j}^{2}}\left(1-\frac{\theta_{j}}{\sin \theta_{j}}\right), \\
& \beta_{1}=\frac{1}{\theta_{j+1}^{2}}\left(1-\frac{\cot \theta_{j+1}}{\theta_{j+1}}\right), \quad \beta_{2}=\frac{1}{\theta_{j}^{2}}\left(1-\frac{\cot \theta_{j}}{\theta_{j}}\right), \\
& \beta_{3}=\frac{\beta_{2}}{\left(\sigma_{j} \beta_{1}+\beta_{2}\right)}, \quad \beta_{4}=\frac{\beta_{1}}{\left(\sigma_{j} \beta_{1}+\beta_{2}\right)}, \quad \theta_{j}=k h_{j} \quad \text { and } \quad y_{j}=y\left(x_{j}\right) .
\end{aligned}
$$

Now, by the continuity conditions of first derivative, i.e., (ii), we get the following scheme:

$$
y_{j+1}-\left(1+\sigma_{j}\right) y_{j}+\sigma_{j} y_{j-1}=h_{j} h_{j+1}\left(\sigma_{j} \alpha F_{j+1}+\left(\sigma_{j} \beta_{1}+\beta_{2}\right) F_{j}+\gamma F_{j-1}\right)+T_{j}\left(h_{j}\right) .
$$

We observe, as $\theta_{j} \rightarrow 0,\left(\alpha, \beta_{1}, \beta_{2}, \gamma\right) \rightarrow\left(\frac{1}{6}, \frac{1}{3}, \frac{1}{3}, \frac{1}{6}\right)$, scheme (13) reduces into the standard variable-mesh cubic spline scheme

$$
y_{j+1}-\left(1+\sigma_{j}\right) y_{j}+\sigma_{j} y_{j-1}=h_{j} h_{j+1}\left[\frac{\sigma_{j}}{6} F_{j+1}+\frac{\left(\sigma_{j}+1\right)}{3} F_{j}+\frac{1}{6} F_{j-1}\right] .
$$

Now, we consider the following approximations evaluated at the grid points $x_{j}, j=$ $1,2,3, \ldots, N-1$ :

$$
\begin{aligned}
& s_{j}=\sigma_{j}\left(\sigma_{j}+1\right), \\
& \bar{y}_{x_{j+1}}=\frac{\left(1+2 \sigma_{j}\right) y_{j+1}-\left(1+\sigma_{j}\right)^{2} y_{j}+\sigma_{j}^{2} y_{j-1}}{h_{j} s_{j}}, \\
& \bar{y}_{x_{j-1}}=\frac{-y_{j+1}+\left(1+\sigma_{j}\right)^{2} y_{j}-\sigma_{j}\left(2+\sigma_{j}\right) y_{j-1}}{h_{j} s_{j}}, \\
& \bar{y}_{x_{j}}=\frac{y_{j+1}+\left(\sigma_{j}^{2}-1\right) y_{j}-\sigma_{j}^{2} y_{j-1}}{h_{j} s_{j}}, \\
& \bar{F}_{j+1}=F\left(x_{j+1}, y_{j+1}, \bar{y}_{x_{j+1}}\right), \\
& \bar{F}_{j-1}=F\left(x_{j-1}, y_{j-1}, \bar{y}_{x_{j-1}}\right), \\
& \bar{F}_{j}=F\left(x_{j}, y_{j}, \bar{y}_{x_{j}}\right), \\
& \overline{\bar{y}}_{x_{j+1}}=\frac{y_{j+1}-y_{j}}{h_{j+1}}+h_{j+1}\left(\alpha \bar{F}_{j}+\beta_{1} \bar{F}_{j+1}\right), \\
& \overline{\bar{y}}_{x_{j-1}}=\frac{y_{j}-y_{j-1}}{h_{j}}-h_{j}\left(\gamma \bar{F}_{j}+\beta_{2} \bar{F}_{j-1}\right), \\
& \overline{\bar{y}}_{x_{j}}=\frac{y_{j+1} \beta_{3}+y_{j}\left(\sigma_{j}^{2} \beta_{4}-\beta_{3}\right)-y_{j-1} \sigma_{j}^{2} \beta_{4}}{h_{j} \sigma_{j}}-h_{j+1}\left(\alpha \beta_{3} \bar{F}_{j+1}-\gamma \beta_{4} \bar{F}_{j-1}\right), \\
& \overline{\bar{F}}_{j+1}=F\left(x_{j+1}, y_{j+1}, \overline{\bar{y}}_{x_{j+1}}\right), \\
& \overline{\bar{F}}_{j-1}=F\left(x_{j-1}, y_{j-1}, \overline{\bar{y}}_{x_{j-1}}\right), \\
& \overline{\bar{F}}_{j}=F\left(x_{j}, y_{j}, \overline{\bar{y}}_{x_{j}}\right) .
\end{aligned}
$$


Simplifying (10)-(12) and the approximations (16)-(27), for $j=1,2,3, \ldots, N$ we get

$$
\begin{aligned}
& \alpha=\frac{1}{6}+O\left(h_{j}^{2}\right)=\gamma, \\
& \beta_{1}=\frac{1}{3}+O\left(h_{j}^{2}\right)=\beta_{2}, \\
& \bar{y}_{x_{j}}=y_{x_{j}}+\frac{1}{6} \sigma_{j} h_{j}^{2} y_{x x x_{j}}+O\left(h_{j}^{3}\right), \\
& \bar{y}_{x_{j+1}}=y_{x_{j+1}}-\frac{1}{6} \sigma_{j}\left(1+\sigma_{j}\right) h_{j}^{2} y_{x x x_{j}}+O\left(h_{j}^{3}\right), \\
& \bar{y}_{x_{j-1}}=y_{x_{j-1}}-\frac{1}{6}\left(1+\sigma_{j}\right) h_{j}^{2} y_{x x x_{j}}+O\left(h_{j}^{3}\right), \\
& \bar{F}_{j+1}=F_{j+1}-\frac{1}{6} \sigma_{j}\left(1+\sigma_{j}\right) h_{j}^{2} y_{x x x_{j}} G+O\left(h_{j}^{3}\right), \\
& \bar{F}_{j-1}=F_{j-1}-\frac{1}{6}\left(1+\sigma_{j}\right) h_{j}^{2} y_{x x x_{j}} G+O\left(h_{j}^{3}\right), \\
& \bar{F}_{j}=F_{j}+\frac{1}{6} \sigma_{j} h_{j}^{2} y_{x x x} G+O\left(h_{j}^{3}\right), \\
& \overline{\bar{y}}_{x_{j}}=y_{x_{j}}+O\left(h_{j}^{3}\right), \\
& \overline{\bar{y}}_{x_{j \pm 1}}=y_{x_{j \pm 1}}+O\left(h_{j}^{3}\right), \\
& \overline{\bar{F}}_{j}=F_{j}+O\left(h_{j}^{3}\right), \\
& \overline{\bar{F}}_{j \pm 1}=F_{j \pm 1}+O\left(h_{j}^{3}\right), \\
& \text { where } G=\frac{\partial F}{\partial y_{x_{j}} .}
\end{aligned}
$$

Now, using (30)-(39) we generate a family of variable-mesh non-polynomial cubic spline schemes of second- and third-order for different values of $P_{j}, R_{j}$ and $Q_{j}$ in the following scheme:

$$
y_{j+1}-\left(1+\sigma_{j}\right) y_{j}+\sigma_{j} y_{j-1}=h_{j} h_{j+1}\left(P_{j} F_{j+1}+Q_{j} F_{j}+R_{j} F_{j-1}\right)+T_{j}\left(h_{j}\right) .
$$

(I) Second-order scheme. For $P_{j}=\sigma_{j} \alpha, Q_{j}=\left(\sigma_{j} \beta_{1}+\beta_{2}\right), R_{j}=\gamma$, the local truncation error $T_{j}$ is $O\left(h_{j}^{4}\right)$, thus leading to a second-order method. $\theta_{j}$ satisfies the consistency condition $\tan \left(\frac{k h_{j}}{2}\right)+\left(\frac{k h_{j}}{2}\right)=\left(\frac{1}{2}\right)$ and this equation has an infinite number of roots. We can use the smallest positive non-zero root of the equation as the value of $\theta_{j}$ i.e., $k h_{j}=8.986818916$.

(II) Third-order scheme. Again, for the following values of $P_{j}, R_{j}$ and $Q_{j}$, we get the second scheme:

$$
P_{j}=\frac{\left(\sigma_{j}^{2}+\sigma_{j}-1\right)}{12}, \quad Q_{j}=\frac{\left(\sigma_{j}+1\right)\left(\sigma_{j}^{2}+3 \sigma_{j}+1\right)}{12}, \quad R_{j}=\frac{\sigma_{j}\left(1+\sigma_{j}-\sigma_{j}^{2}\right)}{12} .
$$

Also, using (38)-(39) we can easily prove that $T_{j}=O\left(h_{j}^{5}\right)$ and the local truncation error in the case of the second discretization scheme with uniform mesh is $O\left(h^{6}\right)$. 
Note that the coefficients $P_{j}, Q_{j}, R_{j}$ are positive if $\frac{(\sqrt{5}-1)}{2}<\sigma_{j}<\frac{(\sqrt{5}+1)}{2}$, thus satisfying condition of convergence of the scheme [29].

\section{Generalization of the schemes}

We generalize our method for the solution of the system of $M$ non-linear boundary value problems (1). At the grid point $x_{j}, j=1,2, \ldots, N-1$, we use the following approximations and schemes:

$$
\begin{aligned}
& S^{(i)}(x)=a_{j}+b_{j}\left(x-x_{j}\right)+c_{j} \sin \left(k\left(x-x_{j}\right)\right)+d_{j} \cos \left(k\left(x-x_{j}\right)\right), \\
& k>0, x_{j-1} \leq x \leq x_{j}, j=1,2,3, \ldots, N . \\
& s_{j}=\sigma_{j}\left(\sigma_{j}+1\right), \\
& \bar{y}_{x_{j+1}}^{(i)}=\frac{\left(1+2 \sigma_{j}\right) y_{j+1}^{(i)}-\left(1+\sigma_{j}\right)^{2} y_{j}^{(i)}+\sigma_{j}^{2} y_{j-1}^{(i)}}{h_{j} s_{j}}, \\
& \bar{y}_{x_{j-1}}^{(i)}=\frac{-y_{j+1}^{(i)}+\left(1+\sigma_{j}\right)^{2} y_{j}^{(i)}-\sigma_{j}\left(2+\sigma_{j}\right) y_{j-1}^{(i)}}{h_{j} s_{j}}, \\
& \bar{y}_{x_{j}}^{(i)}=\frac{y_{j+1}^{(i)}+\left(\sigma_{j}^{2}-1\right) y_{j}^{(i)}-\sigma_{j}^{2} y_{j-1}^{(i)}}{h_{j} s_{j}}, \\
& \bar{F}_{r}^{(i)}=F^{(i)}\left(x_{r}, y_{r}^{(1)}, y_{r}^{(2)}, \ldots, y_{r}^{(i)}, \ldots, y_{r}^{(M)}, \bar{y}_{x_{r}}^{(1)}, \bar{y}_{x_{r}}^{(2)}, \ldots, \bar{y}_{x_{r}}^{(i)}, \ldots, \bar{y}_{x_{r}}^{(M)}\right), \\
& \overline{\bar{y}}_{x_{j+1}}^{(i)}=\frac{y_{j+1}^{(i)}-y_{j}^{(i)}}{h_{j+1}}+h_{j+1}\left(\alpha \bar{F}_{j}^{(i)}+\beta_{1} \bar{F}_{j+1}^{(i)}\right), \\
& \overline{\bar{y}}_{x_{j-1}}^{(i)}=\frac{y_{j}^{(i)}-y_{j-1}^{(i)}}{h_{j}}-h_{j}\left(\gamma \bar{F}_{j}^{(i)}+\beta_{2} \bar{F}_{j-1}^{(i)}\right), \\
& \overline{\bar{y}}_{x_{j}}^{(i)}=\frac{y_{j+1}^{(i)} \beta_{3}+y_{j}^{(i)}\left(\sigma_{j}^{2} \beta_{4}-\beta_{3}\right)-y_{j-1}^{(i)} \sigma_{j}^{2} \beta_{4}}{h_{j} \sigma_{j}}-h_{j+1}\left(\alpha \beta_{3} \bar{F}_{j+1}^{(i)}-\gamma \beta_{4} \bar{F}_{j-1}^{(i)}\right), \\
& \overline{\bar{F}}_{r}^{(i)}=F^{(i)}\left(x_{r}, y_{r}^{(1)}, y_{r}^{(2)}, \ldots, y_{r}^{(i)}, \ldots, y_{r}^{(M)}, \bar{y}_{x_{r}}^{(1)}, \bar{y}_{x_{r}}^{(2)}, \ldots, \overline{\bar{y}}_{x_{r}}^{(i)}, \ldots, \overline{\bar{y}}_{x_{r}}^{(M)}\right), \\
& y_{j+1}^{(i)}-\left(1+\sigma_{j}\right) y_{j}^{(i)}+\sigma_{j} y_{j-1}^{(i)}=h_{j} h_{j+1}\left(\sigma_{j} \alpha \bar{F}_{j+1}^{(i)}+\left(\sigma_{j} \beta_{1}+\beta_{2}\right) \bar{F}_{j}^{(i)}+\gamma \bar{F}_{j-1}^{(i)}\right)+T_{j}\left(h_{j}\right),
\end{aligned}
$$

and also

$$
y_{j+1}^{(i)}-\left(1+\sigma_{j}\right) y_{j}^{(i)}+\sigma_{j} y_{j-1}^{(i)}=h_{j} h_{j+1}\left(P_{j} \overline{\bar{F}}_{j+1}^{(i)}+Q_{j} \overline{\bar{F}}_{j}^{(i)}+R_{j} \overline{\bar{F}}_{j-1}^{(i)}\right)+T_{j}\left(h_{j}\right),
$$

where

$$
\begin{aligned}
& a_{j}=y_{j}^{(i)}+\frac{F_{j}^{(i)}}{k^{2}}, \quad b_{j}=\frac{y_{j+1}^{(i)}-y_{j}^{(i)}}{h_{j}}+\frac{F_{j+1}^{(i)}-F_{j}^{(i)}}{k^{2} h_{j}}, \\
& c_{j}=-\frac{F_{j}^{(i)}}{k^{2}} \quad \text { and } \quad d_{j}=\frac{F_{j}^{(i)} \cos \left(k h_{j}\right)-F_{j+1}^{(i)}}{k^{2} \sin \left(k h_{j}\right)}, \\
& \alpha=\frac{-1}{\theta_{j+1}^{2}}\left(1-\frac{\theta_{j+1}}{\sin \theta_{j+1}}\right), \quad \gamma=\frac{-1}{\theta_{j}^{2}}\left(1-\frac{\theta_{j}}{\sin \theta_{j}}\right), \\
& \beta_{1}=\frac{1}{\theta_{j+1}^{2}}\left(1-\frac{\cot \theta_{j+1}}{\theta_{j+1}}\right), \quad \beta_{2}=\frac{1}{\theta_{j}^{2}}\left(1-\frac{\cot \theta_{j}}{\theta_{j}}\right),
\end{aligned}
$$




$$
\begin{array}{ll}
\beta_{3}=\frac{\beta_{2}}{\left(\sigma_{j} \beta_{1}+\beta_{2}\right)}, & \beta_{4}=\frac{\beta_{1}}{\left(\sigma_{j} \beta_{1}+\beta_{2}\right)}, \quad \theta_{j}=k h_{j} \quad \text { and } \quad r=j, j \pm 1, \\
P_{j}=\frac{\left(\sigma_{j}^{2}+\sigma_{j}-1\right)}{12}, & Q_{j}=\frac{\left(\sigma_{j}+1\right)\left(\sigma_{j}^{2}+3 \sigma_{j}+1\right)}{12},
\end{array}
$$

\section{Application to fourth-order singular boundary value problem}

We consider a linear fourth-order singular boundary value problem:

$$
\begin{aligned}
& \frac{d^{4} y}{d x^{4}}=F\left(x, y, \frac{d y}{d x}, \frac{d^{2} y}{d x^{2}}, \frac{d^{3} y}{d x^{3}}\right), \quad 0<x \leq b, \\
& \text { subject to } \quad y(0)=A_{1}, \quad y(b)=B_{1}, \quad \frac{d^{2}}{d x^{2}} y(0)=A_{2}, \quad \frac{d^{2}}{d x^{2}} y(b)=B_{2},
\end{aligned}
$$

where $F\left(x, y, \frac{d y}{d x}, \frac{d^{2} y}{d x^{2}}, \frac{d^{3} y}{d x^{3}}\right)=a(x) \frac{d^{3} y}{d x^{3}}+b(x) \frac{d^{2} y}{d x^{2}}+c(x) \frac{d y}{d x}+d(x) y(x)+g(x) ; A_{1}, A_{2}, B_{1}, B_{2}$ are real constants and at least one of the coefficients $a(x), b(x), c(x), d(x)$ or $g(x)$ may be singular at $x=0$. We may rewrite the problem (60)-(61) as a system of second-order boundary value problems:

$$
\begin{aligned}
& \frac{d^{2} y}{d x^{2}}=z(x), \\
& \frac{d^{2} z}{d x^{2}}=a(x) \frac{d z}{d x}+b(x) z(x)+c(x) \frac{d y}{d x}+d(x) y(x)+g(x),
\end{aligned}
$$

subject to

$$
y(0)=A_{1}, \quad z(0)=A_{2}, \quad y(b)=B_{1}, \quad z(b)=B_{2} .
$$

Applying the difference scheme (52) to the coupled second-order boundary value problem (62)-(63), we obtain the following difference scheme:

$$
\begin{aligned}
\sigma_{j} y_{j-1}-\left(1+\sigma_{j}\right) y_{j}+y_{j+1}= & h_{j} h_{j+1}\left(P_{j} z_{j+1}+Q_{j} z_{j}+R_{j} z_{j-1}\right), \\
\sigma_{j} z_{j-1}-\left(1+\sigma_{j}\right) z_{j}+z_{j+1}= & h_{j} h_{j+1}\left[P_{j}\left(a_{j+1} \bar{z}_{x_{j+1}}+b_{j+1} z_{j+1}+c_{j+1} \bar{y}_{x_{j+1}}+d_{j+1} y_{j+1}+g_{j+1}\right)\right. \\
& +Q_{j}\left(a_{j} \bar{z}_{x_{j}}+b_{j} z_{j}+c_{j} \bar{y}_{x_{j}}+d_{j} y_{j}+g_{j}\right) \\
& \left.+R_{j}\left(a_{j-1} \bar{z}_{x_{j-1}}+b_{j-1} z_{j-1}+c_{j-1} \bar{y}_{x_{j-1}}+d_{j-1} y_{j-1}+g_{j-1}\right)\right],
\end{aligned}
$$

where $P_{j}=\sigma_{j} \alpha, Q_{j}=\left(\sigma_{j} \beta_{1}+\beta_{2}\right), R_{j}=\gamma$ and $j=1,2, \ldots, N-1$. The boundary value problem has a singularity at some $x=0$ and hence, the scheme fails at $j=1$. Therefore, we define the following approximations around the $j$ th node to evade the singularity:

$$
\begin{aligned}
& a_{j-1}^{*}=a_{j}-h_{j} a_{x_{j}}+O\left(h_{j}^{2}\right), \\
& a_{j+1}^{*}=a_{j}+\sigma_{j} h_{j} a_{x_{j}}+O\left(h_{j}^{2}\right), \\
& a_{j-1}^{* *}=a_{j}-h_{j} a_{x_{j}}+\frac{\left(h_{j}\right)^{2}}{2} a_{x x_{j}}+O\left(h_{j}^{3}\right), \\
& a_{j+1}^{* *}=a_{j}+\sigma_{j} h_{j} a_{x_{j}}+\frac{\left(\sigma_{j} h_{j}\right)^{2}}{2} a_{x x_{j}}+O\left(h_{j}^{3}\right) .
\end{aligned}
$$


Similar relations for $b_{j \pm 1}, c_{j \pm 1}, d_{j \pm 1}, g_{j \pm 1}$ can also be defined. Now, using equations (67)-(68) in (65)-(66) we get

$$
\begin{aligned}
\sigma_{j} y_{j-1}-\left(1+\sigma_{j}\right) y_{j}+y_{j+1}= & h_{j} h_{j+1}\left(P_{j} z_{j+1}+Q_{j} z_{j}+R_{j} z_{j-1}\right), \\
\sigma_{j} z_{j-1}-\left(1+\sigma_{j}\right) z_{j}+z_{j+1}= & h_{j} h_{j+1}\left[P_{j}\left(a_{j+1}^{*} \bar{z}_{x_{j+1}}+b_{j+1}^{*} z_{j+1}+c_{j+1}^{*} \bar{y}_{x_{j+1}}+d_{j+1}^{*} y_{j+1}+g_{j+1}^{*}\right)\right. \\
& +Q_{j}\left(a_{j} \bar{z}_{x_{j}}+b_{j} z_{j}+c_{j} \bar{y}_{x_{j}}+d_{j} y_{j}+g_{j}\right) \\
& \left.+R_{j}\left(a_{j-1}^{*} \bar{z}_{x_{j-1}}+b_{j-1}^{*} z_{j-1}+c_{j-1}^{*} \bar{y}_{x_{j-1}}+d_{j-1}^{*} y_{j-1}+g_{j-1}^{*}\right)\right]
\end{aligned}
$$

$$
\text { for } j=1, \ldots, N-1 \text {. }
$$

Finally, substituting (44)-(46) in (71)-(72), we obtain the vector difference equation of boundary value problem (60)-(61) as follows:

$$
\left[\begin{array}{ll}
\operatorname{sub}_{j}^{11} & \operatorname{sub}_{j}^{12} \\
\operatorname{sub}_{j}^{21} & \operatorname{sub}_{j}^{22}
\end{array}\right]\left[\begin{array}{c}
y_{j-1} \\
z_{j-1}
\end{array}\right]+\left[\begin{array}{ll}
\operatorname{diag}_{j}^{11} & \operatorname{diag}_{j}^{12} \\
\operatorname{diag}_{j}^{21} & \operatorname{diag}_{j}^{22}
\end{array}\right]\left[\begin{array}{c}
y_{j} \\
z_{j}
\end{array}\right]+\left[\begin{array}{ll}
\sup _{j}^{11} & \sup _{j}^{12} \\
\sup _{j}^{21} & \sup _{j}^{22}
\end{array}\right]\left[\begin{array}{c}
y_{j+1} \\
z_{j+1}
\end{array}\right]=\left[\begin{array}{c}
\phi_{j}^{1} \\
\phi_{j}^{2}
\end{array}\right],
$$

where

$$
\begin{aligned}
& \operatorname{sub}_{j}^{11}=-\sigma_{j}, \quad \operatorname{sub}_{j}^{12}=h_{j}^{2} \sigma_{j} P_{j}, \\
& \operatorname{sub}_{j}^{21}=\frac{h_{j} c_{j}}{\left(\sigma_{j}+1\right)}\left[\left(P_{j}-Q_{j}\right) \sigma_{j}^{2}-R_{j} \sigma_{j}\left(2+\sigma_{j}\right)\right]+h_{j}^{2}\left[\frac{c_{x_{j}}\left(P_{j} \sigma_{j}^{2}+R_{j} \sigma_{j}\left(2+\sigma_{j}\right)\right)}{\left(1+\sigma_{j}\right)}+R_{j} \sigma_{j} d_{j}\right] \\
& +h_{j}^{3}\left(-\sigma_{j} d_{x_{j}} R_{j}\right) \text {, } \\
& \operatorname{sub}_{j}^{22}=-\sigma_{j}+\frac{h_{j} a_{j}}{\left(\sigma_{j}+1\right)}\left[\left(P_{j}-Q_{j}\right) \sigma_{j}^{2}-R_{j} \sigma_{j}\left(2+\sigma_{j}\right)\right] \\
& +h_{j}^{2}\left[\frac{a_{x_{j}}\left(P_{j} \sigma_{j}^{2}+R_{j} \sigma_{j}\left(2+\sigma_{j}\right)\right)}{\left(1+\sigma_{j}\right)}+R_{j} \sigma_{j} b_{j}\right] \\
& +h_{j}^{3}\left(-\sigma_{j} b_{x_{j}} R_{j}\right) \text {, } \\
& \operatorname{diag}_{j}^{11}=\left(1+\sigma_{j}\right), \quad \operatorname{diag}_{j}^{12}=\frac{h_{j}^{2} \sigma_{j}\left(\sigma_{j}+1\right)}{6}, \\
& \operatorname{diag}_{j}^{21}=\frac{h_{j} c_{j}}{\left(\sigma_{j}+1\right)}\left[\left(-P_{j}+R_{j}\right)\left(1+\sigma_{j}\right)^{2}+Q_{j}\left(\sigma_{j}^{2}-1\right)\right]+h_{j}^{2}\left[c_{x_{j}}\left(-P_{j} \sigma_{j}-R_{j}\right)\left(1+\sigma_{j}\right)+Q_{j} \sigma_{j} d_{j}\right], \\
& \operatorname{diag}_{j}^{22}=\left(1+\sigma_{j}\right)+\frac{h_{j} a_{j}}{\left(\sigma_{j}+1\right)}+\left[\left(-P_{j}+R_{j}\right)\left(1+\sigma_{j}\right)^{2}+Q_{j}\left(\sigma_{j}^{2}-1\right)\right] \\
& +h_{j}^{2}\left[a_{x_{j}}\left(-P_{j} \sigma_{j}-R_{j}\right)\left(1+\sigma_{j}\right)+Q_{j} \sigma_{j} b_{j}\right] \text {, } \\
& \sup _{j}^{11}=-1, \quad \sup _{j}^{12}=h_{j}^{2} R_{j}, \\
& \sup _{j}^{21}=\frac{h_{j} c_{j}}{\left(\sigma_{j}+1\right)}\left[P_{j}\left(2 \sigma_{j}+1\right)-R_{j}+Q_{j}\right] \\
& +h_{j}^{2}\left[\frac{c_{x_{j}}\left(P_{j}\left(1+2 \sigma_{j}\right) \sigma_{j}+R_{j}\right)}{\left(1+\sigma_{j}\right)}+P_{j} \sigma_{j} d_{j}\right]+h_{j}^{3}\left(\sigma_{j}^{2} d_{x_{j}} R_{j}\right), \\
& \sup _{j}^{22}=-1+\frac{h_{j} a_{j}}{\left(\sigma_{j}+1\right)}\left[P_{j}\left(2 \sigma_{j}+1\right)-R_{j}+Q_{j}\right]
\end{aligned}
$$




$$
\begin{array}{r}
+h_{j}^{2}\left[\frac{a_{x_{j}}\left(P_{j}\left(1+2 \sigma_{j}\right) \sigma_{j}+R_{j}\right)}{\left(1+\sigma_{j}\right)}+P_{j} \sigma_{j} b_{j}\right]+h_{j}^{3}\left(\sigma_{j}^{2} b_{x_{j}} P_{j}\right), \\
\phi_{j}^{1}=0, \quad \phi_{j}^{2}=-h_{j}^{2}\left[\sigma_{j}\left(P_{j}+Q_{j}+R_{j}\right) g_{j}+h_{j} \sigma_{j}\left(P_{j} \sigma_{j}-R_{j}\right) g_{x_{j}}\right] .
\end{array}
$$

Similarly, using (43)-(51) and (69)-(70) up to $O\left(h_{j}^{5}\right)$ terms in scheme (53) we get the second difference scheme of higher order.

\subsection{Application to sixth-order singular boundary value problem}

Let us consider a linear singular sixth-order boundary value problem of the following form:

$$
\begin{aligned}
\frac{d^{6} y}{d x^{6}}= & a(x) \frac{d^{5} y}{d x^{5}}+b(x) \frac{d^{4} y}{d x^{4}} \\
& +c(x) \frac{d^{3} y}{d x^{3}}+d(x) \frac{d^{2} y}{d x^{2}}+e(x) \frac{d y}{d x}+f(x) y(x)+g(x), \quad 0<x \leq b,
\end{aligned}
$$

subject to boundary conditions:

$$
\begin{array}{lll}
y(0)=A_{1}, & \frac{d^{2}}{d x^{2}} y(0)=A_{2}, & \frac{d^{4}}{d x^{4}} y(0)=A_{3}, \\
y(b)=B_{1}, & \frac{d^{2}}{d x^{2}} y(b)=B_{2}, & \frac{d^{4}}{d x^{4}} y(b)=B_{3},
\end{array}
$$

where $A_{1}, A_{2}, A_{3}, B_{1}, B_{2}, B_{3}$ are real constants and any one of the coefficients $a(x), b(x)$, $c(x), d(x), e(x), f(x)$ or $g(x)$ may be singular at $x=0$. We may rewrite the problem (74)-(75) as a system of second-order boundary value problems:

$$
\begin{aligned}
& \frac{d^{2} y}{d x^{2}}=z(x), \\
& \frac{d^{2} z}{d x^{2}}=v(x) \\
& \frac{d^{2} v}{d x^{2}}=a(x) \frac{d v}{d x}+b(x) v(x)+c(x) \frac{d z}{d x}+d(x) z(x)+e(x) \frac{d y}{d x}+f(x) y(x)+g(x),
\end{aligned}
$$

subject to

$$
\begin{array}{lll}
y(0)=A_{1}, & z(0)=A_{2}, & v(0)=A_{3}, \\
y(b)=B_{1}, & z(b)=B_{2}, & v(b)=B_{3} .
\end{array}
$$

Applying the difference scheme (52) to the coupled second-order boundary value problem (76)-(78), we obtain the following difference scheme:

$$
\begin{aligned}
\sigma_{j} y_{j-1}-\left(1+\sigma_{j}\right) y_{j}+y_{j+1}= & h_{j} h_{j+1}\left(P_{j} z_{j+1}+Q_{j} z_{j}+R_{j} z_{j-1}\right), \\
\sigma_{j} z_{j-1}-\left(1+\sigma_{j}\right) z_{j}+z_{j+1}= & h_{j} h_{j+1}\left(P_{j} v_{j+1}+Q_{j} v_{j}+R_{j} v_{j-1}\right) \\
\sigma_{j} v_{j-1}-\left(1+\sigma_{j}\right) v_{j}+v_{j+1}= & h_{j} h_{j+1}\left[P _ { j } \left(a_{j+1} \bar{v}_{x_{j+1}}+b_{j+1} v_{j+1}+c_{j+1} \bar{z}_{x_{j+1}}\right.\right. \\
& \left.+d_{j+1} z_{j+1}+e_{j+1} \bar{y}_{x_{j+1}}+f_{j+1} y_{j+1}+g_{j+1}\right) \\
& +Q_{j}\left(a_{j} \bar{v}_{x_{j}}+b_{j} v_{j}+c_{j} \bar{z}_{x_{j}}+d_{j} z_{j}+e_{j} \bar{y}_{x_{j}}+f_{j} y_{j}+g_{j}\right)
\end{aligned}
$$




$$
\begin{aligned}
& +R_{j}\left(a_{j-1} \bar{v}_{x_{j-1}}+b_{j-1} v_{j-1}+c_{j-1} \bar{z}_{x_{j-1}}\right. \\
& \left.\left.+d_{j-1} z_{j-1}+e_{j-1} \bar{y}_{x_{j-1}}+f_{j-1} y_{j-1}+g_{j-1}\right)\right],
\end{aligned}
$$

where $P_{j}, Q_{j}, R_{j}$ are defined in Section 2 . The boundary value problem has a singularity at $x=0$. Therefore, as in Section 4 we use the approximation (67)-(68) in (80)-(82) and we get

$$
\begin{aligned}
\sigma_{j} y_{j-1}-\left(1+\sigma_{j}\right) y_{j}+y_{j+1}= & h_{j} h_{j+1}\left(P_{j} z_{j+1}+Q_{j} z_{j}+R_{j} z_{j-1}\right), \\
\sigma_{j} z_{j-1}-\left(1+\sigma_{j}\right) z_{j}+z_{j+1}= & h_{j} h_{j+1}\left(P_{j} v_{j+1}+Q_{j} v_{j}+R_{j} v_{j-1}\right), \\
\sigma_{j} v_{j-1}-\left(1+\sigma_{j}\right) v_{j}+v_{j+1}= & h_{j} h_{j+1}\left[P _ { j } \left(a_{j+1}^{*} \bar{x}_{x_{j+1}}+b_{j+1}^{*} v_{j+1}+c_{j+1}^{*} \bar{z}_{x_{j+1}}\right.\right. \\
& \left.+d_{j+1}^{*} z_{j+1}+e_{j+1}^{*} \bar{y}_{x_{j+1}}+f_{j+1}^{*} y_{j+1}+g_{j+1}^{*}\right) \\
& +Q_{j}\left(a_{j} \bar{v}_{x_{j}}+b_{j} v_{j}+c_{j} \bar{z}_{x_{j}}+d_{j} z_{j}+e_{j} \bar{y}_{x_{j}}+f_{j} y_{j}+g_{j}\right) \\
& +R_{j}\left(a_{j-1}^{*} \bar{v}_{x_{j-1}}+b_{j-1}^{*} v_{j-1}+c_{j-1}^{*} \bar{z}_{x_{j-1}}\right. \\
& \left.\left.+d_{j-1}^{*} z_{j-1}+e_{j-1}^{*} \bar{y}_{x_{j-1}}+f_{j-1}^{*} y_{j-1}+g_{j-1}^{*}\right)\right] .
\end{aligned}
$$

Finally, substituting (43)-(47) in (83)-(85), we obtain the vector difference equation of boundary value problem (74) as follows:

$$
\begin{aligned}
& {\left[\begin{array}{lll}
\operatorname{sub}_{j}^{11} & \operatorname{sub}_{j}^{12} & \operatorname{sub}_{j}^{13} \\
\operatorname{sub}_{j}^{21} & \operatorname{sub}_{j}^{22} & \operatorname{sub}_{j}^{23} \\
\operatorname{sub}_{j}^{31} & \operatorname{sub}_{j}^{32} & \operatorname{sub}_{j}^{33}
\end{array}\right]\left[\begin{array}{l}
y_{j-1} \\
z_{j-1} \\
v_{j-1}
\end{array}\right]+\left[\begin{array}{lll}
\operatorname{diag}_{j}^{11} & \operatorname{diag}_{j}^{12} & \operatorname{diag}_{j}^{13} \\
\operatorname{diag}_{j}^{21} & \operatorname{diag}_{j}^{22} & \operatorname{diag}_{j}^{23} \\
\operatorname{diag}_{j}^{31} & \operatorname{diag}_{j}^{32} & \operatorname{diag}_{j}^{33}
\end{array}\right]\left[\begin{array}{c}
y_{j} \\
z_{j} \\
v_{j}
\end{array}\right]} \\
& \quad+\left[\begin{array}{lll}
\sup _{j}^{11} & \sup _{j}^{12} & \sup _{j}^{13} \\
\sup _{j}^{21} & \sup _{j}^{22} & \sup _{j}^{23} \\
\sup _{j}^{31} & \sup _{j}^{32} & \sup _{j}^{33}
\end{array}\right]\left[\begin{array}{c}
y_{j+1} \\
z_{j+1} \\
v_{j+1}
\end{array}\right]=\left[\begin{array}{c}
\phi_{j}^{1} \\
\phi_{j}^{2} \\
\phi_{j}^{3}
\end{array}\right],
\end{aligned}
$$

where

$$
\begin{aligned}
& \left\{\begin{array}{l}
\operatorname{sub}_{j}^{11}=-\sigma_{j}, \quad \operatorname{sub}_{j}^{12}=h_{j}^{2} \sigma_{j} R_{j}, \quad \operatorname{sub}_{j}^{13}=0, \\
\operatorname{sub}_{j}^{21}=0, \quad \operatorname{sub}_{j}^{22}=-\sigma_{j}, \quad \operatorname{sub}_{j}^{23}=h_{j}^{2} \sigma_{j} R_{j}, \\
\operatorname{sub}_{j}^{31}=C_{3}, \quad \operatorname{sub}_{j}^{32}=B_{3}, \quad \operatorname{sub}_{j}^{33}=-\sigma_{j}+A_{3},
\end{array}\right. \\
& \left\{\begin{array}{l}
\operatorname{diag}_{j}^{11}=\left(1+\sigma_{j}\right), \quad \operatorname{diag}_{j}^{12}=\frac{h_{j}^{2} \sigma_{j}\left(\sigma_{j}+1\right)}{6}, \quad \operatorname{diag}_{j}^{13}=0, \\
\operatorname{diag}_{j}^{21}=0, \quad \operatorname{diag}_{j}^{22}=\left(1+\sigma_{j}\right), \quad \operatorname{diag}_{j}^{23}=\frac{h_{j}^{2} \sigma_{j}\left(\sigma_{j}+1\right)}{6}, \\
\operatorname{diag}_{j}^{31}=C_{2}, \quad \operatorname{diag}_{j}^{32}=B_{2}, \quad \operatorname{diag}_{j}^{33}=1+\sigma_{j}+A_{2},
\end{array}\right. \\
& \left\{\begin{array}{l}
\sup _{j}^{11}=-1, \quad \sup _{j}^{12}=h_{j}^{2} \sigma_{j}^{2} P_{j}, \quad \sup _{j}^{13}=0, \\
\sup _{j}^{21}=0, \quad \sup _{j}^{22}=-1, \quad \sup _{j}^{23}=h_{j}^{2} \sigma_{j}^{2} P_{j}, \\
\sup _{j}^{31}=C_{1}, \quad \sup _{j}^{32}=B_{1}, \quad \sup _{j}^{33}=-1+A_{1}, \\
A_{1}=\frac{h_{j} a_{j}}{S_{j}}\left(\sigma_{j}^{2} \alpha\left(1+2 \sigma_{j}\right)+\sigma_{j}\left(\sigma_{j} \beta_{1}+\beta_{2}\right)-\sigma_{j} \gamma\right)+h_{j}^{2}\left(\frac{a_{x_{j}}}{S_{j}}\left(\sigma_{j}^{3} \alpha\left(1+2 \sigma_{j}\right)+\sigma_{j} \gamma\right)+\sigma_{j}^{2} \alpha b_{j}\right)
\end{array}\right. \\
& +h_{j}^{3}\left(\frac{a_{x x_{j}}}{2 S_{j}}\left(\sigma_{j}^{4} \alpha\left(1+2 \sigma_{j}\right)-\sigma_{j} \gamma\right)+\sigma_{j}^{2} \alpha b_{x_{j}}\right)+h_{j}^{4}\left(\sigma_{j}^{4} \alpha \frac{b_{x x_{j}}}{2}\right),
\end{aligned}
$$




$$
\begin{aligned}
& A_{2}=\frac{h_{j} a_{j}}{S_{j}}\left(-\sigma_{j}^{2} \alpha\left(1+\sigma_{j}\right)^{2}+\sigma_{j}\left(\sigma_{j} \beta_{1}+\beta_{2}\right)\left(\sigma_{j}^{2}-1\right)+\sigma_{j} \gamma\left(1+\sigma_{j}\right)^{2}\right) \\
& +h_{j}^{2}\left(\frac{a_{x_{j}}\left(1+\sigma_{j}\right)^{2}}{S_{j}}\left(-\sigma_{j}^{3} \alpha-\sigma_{j} \gamma\right)+\sigma_{j}\left(\sigma_{j} \beta_{1}+\beta_{2}\right) b_{j}\right) \\
& +h_{j}^{3}\left(\frac{a_{x x_{j}}\left(1+\sigma_{j}\right)^{2}}{2 S_{j}}\left(-\sigma_{j}^{4} \alpha+\gamma \sigma_{j}\right)\right) \text {, } \\
& A_{3}=\frac{h_{j} a_{j}}{S_{j}}\left(\sigma_{j}^{4} \alpha-\sigma_{j}^{3}\left(\sigma_{j} \beta_{1}+\beta_{2}\right)-\sigma_{j}^{2} \gamma\left(2+\sigma_{j}\right)\right)+h_{j}^{2}\left(\frac{a_{x_{j}}}{S_{j}}\left(\sigma_{j}^{5} \alpha+\sigma_{j}^{2} \gamma\left(2+\sigma_{j}\right)\right)-\sigma_{j} \gamma b_{j}\right) \\
& +h_{j}^{3}\left(\frac{a_{x x_{j}}}{2 S_{j}}\left(\sigma_{j}^{6} \alpha-\sigma_{j}^{2} \gamma\left(2+\sigma_{j}\right)\right)-\sigma_{j} \gamma b_{x_{j}}\right)+h_{j}^{4}\left(\sigma_{j}^{2} \alpha \frac{b_{x x_{j}}}{2}\right) \\
& B_{1}=\frac{h_{j} c_{j}}{S_{j}}\left(\sigma_{j}^{2} \alpha\left(1+2 \sigma_{j}\right)+\sigma_{j}\left(\sigma_{j} \beta_{1}+\beta_{2}\right)-\sigma_{j} \gamma\right)+h_{j}^{2}\left(\frac{c_{x_{j}}}{S_{j}}\left(\sigma_{j}^{3} \alpha\left(1+2 \sigma_{j}\right)+\sigma_{j} \gamma\right)+\sigma_{j}^{2} \alpha d_{j}\right) \\
& +h_{j}^{3}\left(\frac{c_{x x_{j}}}{2 S_{j}}\left(\sigma_{j}^{4} \alpha\left(1+2 \sigma_{j}\right)-\sigma_{j} \gamma\right)+\sigma_{j}^{2} \alpha d_{x_{j}}\right)+h_{j}^{4}\left(\sigma_{j}^{4} \alpha \frac{d_{x x_{j}}}{2}\right) \text {, } \\
& B_{2}=\frac{h_{j} c_{j}}{S_{j}}\left(-\sigma_{j}^{2} \alpha\left(1+\sigma_{j}\right)^{2}+\sigma_{j}\left(\sigma_{j} \beta_{1}+\beta_{2}\right)\left(\sigma_{j}^{2}-1\right)+\sigma_{j} \gamma\left(1+\sigma_{j}\right)^{2}\right) \\
& +h_{j}^{2}\left(\frac{c_{x_{j}}\left(1+\sigma_{j}\right)^{2}}{S_{j}}\left(-\sigma_{j}^{3} \alpha-\sigma_{j} \gamma\right)+\sigma_{j}\left(\sigma_{j} \beta_{1}+\beta_{2}\right) d_{j}\right) \\
& +h_{j}^{3}\left(\frac{c_{x x_{j}}\left(1+\sigma_{j}\right)^{2}}{2 S_{j}}\left(-\sigma_{j}^{4} \alpha+\gamma \sigma_{j}\right)\right) \\
& B_{3}=\frac{h_{j} c_{j}}{S_{j}}\left(\sigma_{j}^{4} \alpha-\sigma_{j}^{3}\left(\sigma_{j} \beta_{1}+\beta_{2}\right)-\sigma_{j}^{2} \gamma\left(2+\sigma_{j}\right)\right)+h_{j}^{2}\left(\frac{a_{x_{j}}}{S_{j}}\left(\sigma_{j}^{5} \alpha+\sigma_{j}^{2} \gamma\left(2+\sigma_{j}\right)\right)-\sigma_{j} \gamma d_{j}\right) \\
& +h_{j}^{3}\left(\frac{a_{x x_{j}}}{2 S_{j}}\left(\sigma_{j}^{6} \alpha-\sigma_{j}^{2} \gamma\left(2+\sigma_{j}\right)\right)-\sigma_{j} \gamma d_{x_{j}}\right)+h_{j}^{4}\left(\sigma_{j}^{2} \alpha \frac{b_{x x_{j}}}{2}\right) \\
& C_{1}=\frac{h_{j} e_{j}}{S_{j}}\left(\sigma_{j}^{2} \alpha\left(1+2 \sigma_{j}\right)+\sigma_{j}\left(\sigma_{j} \beta_{1}+\beta_{2}\right)-\sigma_{j} \gamma\right)+h_{j}^{2}\left(\frac{e_{x_{j}}}{S_{j}}\left(\sigma_{j}^{3} \alpha\left(1+2 \sigma_{j}\right)+\sigma_{j} \gamma\right)+\sigma_{j}^{2} \alpha f_{j}\right) \\
& +h_{j}^{3}\left(\frac{e_{x x_{j}}}{2 S_{j}}\left(\sigma_{j}^{4} \alpha\left(1+2 \sigma_{j}\right)-\sigma_{j} \gamma\right)+\sigma_{j}^{2} \alpha f_{x_{j}}\right)+h_{j}^{4}\left(\sigma_{j}^{4} \alpha \frac{f_{x x_{j}}}{2}\right), \\
& C_{2}=\frac{h_{j} e_{j}}{S_{j}}\left(-\sigma_{j}^{2} \alpha\left(1+\sigma_{j}\right)^{2}+\sigma_{j}\left(\sigma_{j} \beta_{1}+\beta_{2}\right)\left(\sigma_{j}^{2}-1\right)+\sigma_{j} \gamma\left(1+\sigma_{j}\right)^{2}\right) \\
& +h_{j}^{2}\left(\frac{e_{x_{j}}\left(1+\sigma_{j}\right)^{2}}{S_{j}}\left(-\sigma_{j}^{3} \alpha-\sigma_{j} \gamma\right)+\sigma_{j}\left(\sigma_{j} \beta_{1}+\beta_{2}\right) f_{j}\right) \\
& +h_{j}^{3}\left(\frac{e_{x x_{j}}\left(1+\sigma_{j}\right)^{2}}{2 S_{j}}\left(-\sigma_{j}^{4} \alpha+\gamma \sigma_{j}\right)\right) \\
& C_{3}=\frac{h_{j} e_{j}}{S_{j}}\left(\sigma_{j}^{4} \alpha-\sigma_{j}^{3}\left(\sigma_{j} \beta_{1}+\beta_{2}\right)-\sigma_{j}^{2} \gamma\left(2+\sigma_{j}\right)\right)+h_{j}^{2}\left(\frac{e_{x_{j}}}{S_{j}}\left(\sigma_{j}^{5} \alpha+\sigma_{j}^{2} \gamma\left(2+\sigma_{j}\right)\right)-\sigma_{j} \gamma f_{j}\right) \\
& +h_{j}^{3}\left(\frac{e_{x x_{j}}}{2 S_{j}}\left(\sigma_{j}^{6} \alpha-\sigma_{j}^{2} \gamma\left(2+\sigma_{j}\right)\right)-\sigma_{j} \gamma f_{x_{j}}\right)+h_{j}^{4}\left(\sigma_{j}^{2} \alpha \frac{f_{x x_{j}}}{2}\right) \\
& \phi_{j}^{1}=0, \quad \phi_{j}^{2}=0, \\
& \phi_{j}^{3}=-h_{j}^{2} \sigma_{j}\left[\left(\sigma_{j} \alpha+\left(\sigma_{j} \beta_{1}+\beta_{2}\right)+\gamma\right) g_{j}+h_{j}\left(\sigma_{j}^{2} \alpha-\gamma\right) g_{x_{j}}+h_{j}^{2}\left(\sigma_{j}^{3} \alpha+\gamma\right) \frac{g_{x x_{j}}}{2}\right] .
\end{aligned}
$$




\section{Convergence analysis}

We provide the vector convergence analysis for $M=2$, i.e., a fourth-order non-linear singular boundary value problem (60)-(61). We apply the difference scheme (53) to the boundary value problem and obtain the following difference scheme:

$$
\begin{aligned}
& \sigma y_{j-1}-(1+\sigma) y_{j}+y_{j+1}=h_{j}^{2}\left[P_{j} \overline{\bar{z}}_{j+1}+Q_{j} \overline{\bar{z}}_{j}+R_{j} \overline{\bar{z}}_{j-1}\right]+T_{1}\left(h_{j}\right), \\
& \sigma z_{j-1}-(1+\sigma) z_{j}+z_{j+1}=h_{j}^{2}\left[P_{j} \overline{\bar{F}}_{j+1}+Q_{j} \overline{\bar{F}}_{j}+R_{j} \overline{\bar{F}}_{j-1}\right]+T_{2}\left(h_{j}\right),
\end{aligned}
$$

where

$$
P_{j}=\frac{\left(\sigma_{j}^{2}+\sigma_{j}-1\right)}{12}, \quad Q_{j}=\frac{\left(\sigma_{j}+1\right)\left(\sigma_{j}^{2}+3 \sigma_{j}+1\right)}{12}, \quad R_{j}=\frac{\sigma_{j}\left(1+\sigma_{j}-\sigma_{j}^{2}\right)}{12} .
$$

Now, let $\hat{\mathbf{y}}=\left(\hat{y}_{1}, \hat{y}_{2}, \ldots, \hat{y}_{N-1}\right)^{T}, \hat{\mathbf{z}}=\left(\hat{z}_{1}, \hat{z}_{2}, \ldots, \hat{z}_{N-1}\right)^{T}$ represent the exact solutions and $\mathbf{y}=$ $\left(y_{1}, y_{2}, \ldots, y_{N-1}\right)^{T}$ and $\mathbf{z}=\left(z_{1}, z_{2}, \ldots, z_{N-1}\right)^{T}$ be the approximate solutions. Then we define the error as $\hat{\mathbf{y}}-\mathbf{y}=\left(e_{1,1}, e_{2,1}, \ldots, e_{N-1,1}\right)^{T}$ and $\hat{\mathbf{z}}-\mathbf{z}=\left(e_{1,2}, e_{2,2}, \ldots, e_{N-1,2}\right)^{T}$. Next, we define the following approximation:

$$
\begin{aligned}
& \hat{\bar{F}}_{j \pm 1}=\overline{\bar{F}}_{j \pm 1}+e_{j \pm 1,1} G_{j \pm 1}^{1}+\left(\hat{\bar{y}}_{x_{j \pm 1}}-\bar{y}_{x_{j \pm 1}}\right) H_{j \pm 1}^{1}+e_{j \pm 1,2} V_{j \pm 1}^{1}+\left(\hat{\bar{z}}_{x_{j \pm 1}}-\bar{z}_{x_{j \pm 1}}\right) W_{j \pm 1}^{1}, \\
& \hat{\overline{\bar{F}}}_{j \pm 1}=\overline{\bar{F}}_{j \pm 1}+e_{j \pm 1,1} G_{j \pm 1}^{2}+\left(\hat{\overline{\bar{y}}}_{x_{j \pm 1}}-\overline{\bar{y}}_{x_{j \pm 1}}\right) H_{j \pm 1}^{2}+e_{j \pm 1,2} V_{j \pm 1}^{2}+\left(\hat{\overline{\bar{z}}}_{x_{j \pm 1}}-\overline{\bar{z}}_{x_{j \pm 1}}\right) W_{j \pm 1}^{2}, \\
& \hat{\bar{F}}_{j}=\bar{F}_{j}+e_{j, 1} G_{j}^{1}+\left(\hat{\overline{\bar{y}}}_{x_{j}}-\bar{y}_{x_{j}}\right) H_{j}^{1}+e_{j, 2} V_{j}^{2}+\left(\hat{\bar{z}}_{x_{j}}-\bar{z}_{x_{j}}\right) W_{j}^{2}, \\
& \hat{\overline{\bar{F}}}_{j}=\overline{\bar{F}}_{j \pm 1}+e_{j, 1} G_{j}^{1}+\left(\hat{\bar{y}}_{x_{j}}-\overline{\bar{y}}_{x_{j}}\right) H_{j}^{1}+e_{j, 2} V_{j}^{2}+\left(\hat{\overline{\bar{z}}}_{x_{j}}-\overline{\bar{z}}_{x_{j}}\right) W_{j}^{2},
\end{aligned}
$$

where

$$
\begin{array}{lll}
G_{r}^{1}=\frac{\partial \bar{F}}{\partial y_{r}}, & H_{r}^{1}=\frac{\partial \bar{F}}{\partial y_{x_{r}}}, & V_{r}^{1}=\frac{\partial \bar{F}}{\partial z_{r}},
\end{array}
$$

Further, a singularity is at $x=0$. Therefore, we may define $G_{j \pm 1}^{k}, k=1,2$, as the following approximation. Moreover, similar approximations can be also defined for $H_{j \pm 1}^{k}, V_{j \pm 1}^{k}$ and $W_{j \pm 1}^{k}, k=1,2$. We have

$$
\begin{aligned}
& G_{j+1}^{k}=G_{j}^{k}+h_{j} \sigma G_{x j}^{k}+\frac{\left(h_{j} \sigma\right)^{2}}{2} G_{x x_{j}}{ }^{k}, \\
& G_{j-1}^{k}=G_{j}^{k}-h_{j} G_{x j}{ }^{k}+\frac{h_{j}^{2}}{2} G_{x x_{j}}{ }^{k} .
\end{aligned}
$$

Thus, as we use the approximations (44)-(47), (105)-(106) in equations (97)-(98), we get the error equation in matrix form as follows:

$$
L E+T\left(h_{j}\right)=0,
$$


where $E=\left(\left(e_{1,1}, e_{1,2}\right),\left(e_{2,1}, e_{2,2}\right), \ldots,\left(e_{N-1,1}, e_{N-1,2}\right)\right)^{T}, \quad T\left(h_{j}\right)=\left(\left(T_{1}\left(h_{1}\right), T_{2}\left(h_{1}\right)\right),\left(T_{1}\left(h_{2}\right)\right.\right.$, $\left.\left.T_{2}\left(h_{2}\right)\right), \ldots,\left(T_{1}\left(h_{N-1}\right), T_{2}\left(h_{N-1}\right)\right)\right)^{T}$, and $L=\left(L_{k, j}\right)_{k, j=1}^{N-1}$ denote the block tri-diagonal matrix. The block elements of $L$ are as follows:

$$
l_{j, j}=\left[\begin{array}{cc}
1+\sigma & h_{j}^{2} Q_{j} \\
B_{1} & 1+\sigma+B_{2}
\end{array}\right], \quad l_{j, j+1}=\left[\begin{array}{cc}
-1 & h_{j}^{2} P_{j} \\
C_{1} & -1+C_{2}
\end{array}\right], \quad l_{j, j-1}=\left[\begin{array}{cc}
-\sigma & h_{j}^{2} R_{j} \\
A_{1} & -\sigma+A_{2}
\end{array}\right],
$$

where

$$
\begin{aligned}
A_{1}= & h_{j}\left(-R_{j} H_{j}^{2}-Q_{j} \sigma \beta_{4} H_{j}^{2}\right)+\left(R_{j} H_{x_{j}}^{2}+R_{j} G_{j}^{2}\right) \\
& +h_{j}^{3}\left(\frac{R_{j}}{2} H_{x x_{j}}^{2}-R_{j} G_{x_{j}}^{2}\right)+h_{j}^{4}\left(\frac{R_{j}}{2} G_{x x_{j}}^{2}\right), \\
A_{2}= & h_{j}\left(-R_{j} W_{j}^{2}-Q_{j} \sigma \beta_{4} W_{j}^{2}\right)+\left(R_{j} W_{x_{j}}^{2}+R_{j} V_{j}^{2}\right) \\
& +h_{j}^{3}\left(\frac{R_{j}}{2} W_{x x_{j}}^{2}-R_{j} V_{x_{j}}^{2}-R_{j} \beta_{2} H_{j}^{2}+Q_{j} \sigma \beta_{4} \gamma H_{j}^{2}\right) \\
& +h_{j}^{4}\left(\frac{R_{j}}{2} V_{x x_{j}}^{2}+R_{j} \beta_{2} H_{j}^{2}\right), \\
B_{1}= & h_{j}\left(\frac{-P_{j} H_{j}^{2}}{\sigma}+R_{j} H_{j}^{2}+Q_{j} \frac{\sigma^{2} \beta_{4}-\beta_{3}}{\sigma} H_{j}^{2}\right) \\
& +h_{j}^{2}\left(-P_{j} H_{x_{j}}^{2}-R_{j} H_{x_{j}}^{2}+Q_{j} G_{j}^{2}\right)+h_{j}^{3}\left(\frac{-P_{j} \sigma+R_{j}}{2} H_{x x_{j}}^{2}\right), \\
B_{2}= & h_{j}\left(\frac{-P_{j} W_{j}^{2}}{\sigma}+R_{j} W_{j}^{2}+Q_{j} \frac{\sigma^{2} \beta_{4}-\beta_{3}}{\sigma} W_{j}^{2}\right)+h_{j}^{2}\left(-P_{j} W_{x_{j}}^{2}-R_{j} W_{x_{j}}^{2}+Q_{j} V_{j}^{2}\right) \\
& +h_{j}^{3}\left(\frac{-P_{j} \sigma+R_{j}}{2} W_{x x_{j}}^{2}\right)+h_{j}^{2}\left(\sigma \alpha P_{j}-R_{j} \gamma\right)+h_{j}^{4}\left(H_{j}^{2}\left(\sigma^{2} \alpha P_{j}-R_{j} \gamma\right)\right), \\
& +h_{j}^{4}\left(\frac{P_{j} \sigma^{2} V_{x x_{j}}^{2}}{2}+P_{j} \sigma^{2} \beta_{1} H_{x_{j}}^{2}\right) . \\
C_{1}= & h_{j}\left(\frac{P_{j}+Q_{j} \beta_{3}}{\sigma} H_{j}^{2}\right)+h_{j}^{2}\left(P_{j} H_{x_{j}}+P_{j} G_{j}^{2}\right) \\
& +h_{j}^{3} P_{j} \sigma\left(\frac{P_{x x_{j}}}{2}+G_{x_{j}}^{2}\right)+h_{j}^{4}\left(\frac{P_{j} \sigma^{2} G_{x x_{j}}^{2}}{2}\right), \\
C_{2}= & h_{j}\left(\frac{P_{j}+Q_{j} \beta_{3}}{\sigma} W_{j}^{2}\right)+h_{j}^{2}\left(P_{j} W_{x_{j}}+P_{j} V_{j}^{2}\right) \\
& \left.\left.Q_{j} \sigma \alpha \beta_{3} H_{j}^{2}+\sigma \beta_{1} P_{j} H_{x_{j}}^{2}\right)\right) \\
& \\
& \\
&
\end{aligned}
$$

Further, for some $K_{1}, K_{2}>0$, we assume

$$
\begin{aligned}
& K_{1}=\max \left(\left|G_{j}^{2}\right|,\left|H_{j}^{2}\right|,\left|G_{x_{j}}^{2}\right|,\left|H_{x_{j}}^{2}\right|,\left|G_{x x_{j}}^{2}\right|,\left|H_{x x_{j}}^{2}\right|\right), \\
& K_{2}=\max \left(\left|V_{j}^{2}\right|,\left|W_{j}^{2}\right|,\left|V_{x_{j}}^{2}\right|,\left|W_{x_{j}}^{2}\right|,\left|V_{x x_{j}}^{2}\right|,\left|W_{x x_{j}}^{2}\right|\right) .
\end{aligned}
$$


Then, using $l_{j, j+1} l_{j, j-1}$ and (114)-(115), we get

$$
\begin{gathered}
\left\|\sup _{j}\right\|_{\infty} \leq \max _{1 \leq j \leq N-2}\left\{\begin{array}{c}
1+h_{j}^{2} P_{j}, \\
1+h_{j}\left(\left(\frac{P_{j}+Q_{j} \beta_{3}}{\sigma}\right)+2 h_{j} P_{j}+h_{j}^{2} \frac{3 P_{j} \sigma}{2}+h_{j}^{3} \frac{P_{j} \sigma_{j}^{2}}{2}\right)\left(\left|K_{1}\right|+\left|K_{2}\right|\right) \\
+h_{j}^{3}\left(\left|P_{j} \beta_{1}-Q \sigma_{j} \alpha \beta_{4}\right|+P_{j} \sigma_{j}^{2} \beta_{1}\right)\left|K_{1}\right|,
\end{array}\right. \\
\left\|\operatorname{sub}_{j}\right\|_{\infty} \leq \max _{1 \leq j \leq N-2}\left\{\begin{array}{c}
\sigma+h_{j}^{2} R_{j}, \\
\sigma+h_{j}\left(\left(R_{j}+Q_{j} \sigma \beta_{4}\right)+2 h_{j} R_{j}+h_{j}^{2} \frac{3 R_{j}}{2}+h_{j}^{3} \frac{R_{j}}{2}\right)\left(\left|K_{1}\right|+\left|K_{2}\right|\right) \\
+h_{j}^{3}\left(\left|R_{j} \beta_{2}-Q \sigma_{j} \gamma \beta_{4}\right|+R_{j} \beta_{2}\right)\left|K_{1}\right| .
\end{array}\right.
\end{gathered}
$$

Thus for sufficiently small $h_{j}$, we get $\left\|\operatorname{sub}_{j}\right\|_{\infty} \leq \sigma$ and $\left\|\sup _{j}\right\|_{\infty} \leq 1$. Hence, $L$ is irreducible. Next we prove that $L$ is monotone. We let the sum of the elements of the $k$ th row of $L$ be $\mathrm{SUM}_{k}$. Then

$$
\begin{aligned}
\operatorname{SUM}_{k}= & \sigma+\frac{h_{j}^{2}}{12}\left(P_{j}+Q_{j}\right), \quad k=1, \\
\operatorname{SUM}_{k}= & \sigma+h_{j} r_{1, j}+h_{j}^{2}\left(\left(P_{j}+Q_{j}\right)\left(G_{j}^{2}+V_{j}^{2}\right)-R\left(H_{x_{j}}^{2}+W_{x_{j}}^{2}\right)\right)+O\left(h_{j}^{3}\right), \quad k=2, \\
\operatorname{SUM}_{k}= & h_{j}^{2}\left(P_{j}+Q_{j}+R_{j}\right), \quad k=3,5, \ldots, N-4, \\
\operatorname{SUM}_{k}= & h_{j}^{2}\left(P_{j}+Q_{j}+R_{j}\right)\left(G_{j}^{2}+V_{j}^{2}\right), \quad k=4,6, \ldots, N-3, \\
\operatorname{SUM}_{k}= & 1+h_{j}^{2}\left(R_{j}+Q_{j}\right), \quad k=N-2, \\
\operatorname{SUM}_{k}= & 1+h_{j}\left(\left(r_{2, j}\right)+h_{j}\left(\left(R_{j}+Q_{j}\right)\left(G_{j}^{2}+V_{j}^{2}\right)\right.\right. \\
& \left.\left.-P_{j}\left(H_{x_{j}}^{2}+W_{x_{j}}^{2}\right)\right)\right)+O\left(h_{j}^{3}\right), \quad k=N-1,
\end{aligned}
$$

where

$$
\begin{aligned}
& r_{1, j}=\left(R_{j}+Q_{j} \sigma \beta_{4}\right)\left(H_{j}^{2}+W_{j}^{2}\right), \\
& r_{2, j}=\frac{-P_{j}-Q_{j} \beta_{3}}{\sigma}\left(H_{j}^{2}+W_{j}^{2}\right) .
\end{aligned}
$$

Now, using (114)-(115), we have

$$
\left|r_{1, j}\right| \leq\left|\left(R_{j}+Q_{j} \sigma \beta_{4}\right)\right|\left(\left|K_{1}\right|+\left|K_{2}\right|\right), \quad\left|r_{2, j}\right|=\left|\frac{P_{j}+Q_{j} \beta_{3}}{\sigma}\right|\left(\left|K_{1}\right|+\left|K_{2}\right|\right) .
$$

Finally, for sufficiently small $h_{j}$ and (116)-(121), we can easily prove that $L$ is monotone. Therefore, $L^{-1}$ exists and $L^{-1} \geq 0$. Hence by (107) we have

$$
\|E\|=\left\|L^{-1}\right\|\|T\|
$$

Now for sufficiently small $h_{j}$ and by (116)-(121) we can say that

$$
\mathrm{SUM}_{k}> \begin{cases}h_{j}^{2}(P+Q), & k=1, \\ h_{j}^{2}\left((P+Q)\left(\left|K_{1}\right|+\left|K_{2}\right|\right)\right), & k=2,\end{cases}
$$




$$
\begin{aligned}
& \operatorname{SUM}_{k} \geq \begin{cases}h_{j}^{2}(P+Q+R), & k=3,5, \ldots, N-4, \\
h_{j}^{2}(P+Q+R)\left(\left|K_{1}\right|+\left|K_{2}\right|\right), & k=4,6, \ldots, N-3,\end{cases} \\
& \mathrm{SUM}_{k}> \begin{cases}h_{j}^{2}(R+Q), & k=N-2, \\
h_{j}^{2}\left((R+Q)\left(\left|K_{1}\right|+\left|K_{2}\right|\right)\right), & k=N-1 .\end{cases}
\end{aligned}
$$

Since $\sigma \neq 0$ we can say that

$$
\begin{aligned}
\operatorname{SUM}_{k} & >\max \left[h_{j}^{2}(P+Q), h_{j}^{2}\left((P+Q)\left(\left|K_{1}\right|+\left|K_{2}\right|\right)\right)\right] \\
& =h_{j}^{2}\left((P+Q)\left(\left|K_{1}\right|+\left|K_{2}\right|\right)\right), \quad \text { for } k=1,2, \\
\operatorname{SUM}_{k} & \geq \max \left[h_{j}^{2}(P+Q+R), h_{j}^{2}(P+Q+R)\left(\left|K_{1}\right|+\left|K_{2}\right|\right)\right] \\
& =h_{j}^{2}(P+Q+R)\left(\left|K_{1}\right|+\left|K_{2}\right|\right), \quad \text { for } k=3,4, \ldots, N-3, \\
\mathrm{SUM}_{k} & >\max \left[h_{j}^{2}(R+Q), h_{j}^{2}(R+Q)\left(\left|K_{1}\right|+\left|K_{2}\right|\right)\right] \\
& =h_{j}^{2}\left((R+Q)\left(\left|K_{1}\right|+\left|K_{2}\right|\right)\right), \quad \text { for } k=N-2, N-1 .
\end{aligned}
$$

Let $L_{i, k}^{-1}$ be the $(i, k)$ th element of $L^{-1}$, then by the theory of matrices [30] for $i=1,2, \ldots, N-1$

$$
L_{i, k}^{-1} \leq \frac{1}{\mathrm{SUM}_{k}} .
$$

By using (128)-(130), we have

$$
\frac{1}{\mathrm{SUM}_{k}} \leq \begin{cases}\frac{1}{h_{j}^{2}(P+Q)\left(\left|K_{1}\right|+\left|K_{2}\right|\right)}, & k=1,2, \\ \frac{1}{h_{j}^{2}(P+Q+R)\left(\left|K_{1}\right|+\left|K_{2}\right|\right)}, & k=3,4,5, \ldots, N-3, \\ \frac{1}{h_{j}^{2}(R+Q)\left(\left|K_{1}\right|+\left|K_{2}\right|\right)}, & k=N-2, N-1 .\end{cases}
$$

Now let us define

$$
\left\|L_{i, k}^{-1}\right\|=\max _{1 \leq i \leq N-1} \sum_{k=1}^{N-1}\left|L_{i, k}^{-1}\right| \quad \text { and } \quad\left\|T_{j}\right\|=\max _{1 \leq j \leq N-1} \sum_{j=1}^{N-1}\left|T_{j}\left(h_{j}\right)\right| .
$$

Therefore, as discussed in Section 2 in scheme (53), $T_{j}\left(h_{j}\right)=O\left(h_{j}^{5}\right)$ and using (124), (131)(133) we get

$$
\|E\| \leq \frac{1}{h_{j}^{2}\left(\left|K_{1}\right|+\left|K_{2}\right|\right)}\left[\frac{1}{(P+Q)}+\frac{1}{(P+Q+R)}+\frac{1}{(R+Q)}\right] O\left(h_{j}^{5}\right)=O\left(h_{j}^{3}\right) .
$$

Hence, the third-order vector convergence of scheme (53) follows. Along similar lines, we can prove the second-order vector convergence of scheme (52) for a system of secondorder boundary value problems (1).

Theorem 1 The scheme (53) for the numerical solution of system of non-linear singular boundary value problem (1) with sufficiently small $h_{j}$ and $\sigma \neq 1$ has third-order convergence under appropriate conditions. 


\section{Numerical illustration}

To illustrate the comparative performance of our method with existing methods, we solved the following eight problems. The root mean square errors (RMSs) in the case of a variable mesh, the maximum absolute error (MA) and the relative error (RE) for a uniform mesh are tabulated in Tables 1-8. Let $h_{1}=\frac{(\sigma-1)}{\left(\sigma^{N}-1\right)}, \sigma \neq 1$. Therefore, the rest of the $h_{j}$ can be obtained: $h_{j+1}=\sigma h_{j}, j=1, \ldots, N-1$. In the case of the presence of a boundary layer near the left or right end of the domain, take

$$
h_{1}= \begin{cases}\frac{\sigma-1}{\sigma^{N}-1}, & \sigma>1, \\ \frac{1-\sigma}{1-\sigma^{N}}, & \sigma<1 .\end{cases}
$$

This ensures the presence of mesh points in the boundary layer region near the left or right end of the interval. The linear system of difference equations have been solved by the block Gauss elimination method and the non-linear system of difference equations by the block Newton's method in which we have considered $y_{0}=0$ as the initial approximation. Also, without loss of generality, throughout we will use $\theta_{j+1}=\theta_{j}=\theta$. This does not affect the accuracy of the scheme. All calculations have been done in Matlab 07.

Table 1 Example 6.1

\begin{tabular}{|c|c|c|c|c|}
\hline \multirow[t]{2}{*}{$N$} & \multicolumn{2}{|l|}{ RMS error } & \multicolumn{2}{|l|}{ MA error } \\
\hline & $\boldsymbol{O}\left(h_{j}^{2}\right)$ method & $O\left(h_{j}^{3}\right)$ method & $\bar{O}\left(h^{4}\right)$ method & Ramadan [31] \\
\hline 8 & $3.6416 \mathrm{e}-03$ & $2.5016 \mathrm{e}-05$ & $2.0053 e-06$ & $3.010 \mathrm{e}-05$ \\
\hline 16 & $1.4662 \mathrm{e}-03$ & $5.5065 \mathrm{e}-06$ & $1.2888 \mathrm{e}-07$ & $1.8318 \mathrm{e}-06$ \\
\hline 32 & $8.1036 e-04$ & $2.2304 \mathrm{e}-06$ & $8.1743 e-09$ & $1.1179 \mathrm{e}-07$ \\
\hline
\end{tabular}

Table 2 Example 6.2

\begin{tabular}{|c|c|c|c|c|c|}
\hline \multirow[t]{2}{*}{$N$} & \multicolumn{2}{|l|}{ RMS error } & \multicolumn{3}{|l|}{ MA error } \\
\hline & $O\left(h_{j}^{2}\right)$ method & $O\left(h_{j}^{3}\right)$ method & $\overline{O\left(h^{4}\right) \text { method }}$ & Akram et al. [21] & Siddiqi et al. [32] \\
\hline 8 & $3.6473 \mathrm{e}-03$ & $2.4393 \mathrm{e}-05$ & $1.9706 \mathrm{e}-06$ & $1.5379 \mathrm{e}-06$ & $8.1514 \mathrm{e}-05$ \\
\hline 16 & $1.4829 \mathrm{e}-03$ & $5.3686 \mathrm{e}-06$ & $1.2665 \mathrm{e}-07$ & 1.9790 e-07 & $2.1052 \mathrm{e}-05$ \\
\hline 32 & $8.2427 e-04$ & $2.1736 \mathrm{e}-06$ & $8.0345 e-09$ & $4.0596 \mathrm{e}-08$ & $5.3084 \mathrm{e}-06$ \\
\hline
\end{tabular}

Table 3 Example 6.3

\begin{tabular}{|c|c|c|c|c|c|}
\hline \multirow[t]{2}{*}{$\mathbf{N}$} & \multicolumn{2}{|l|}{ RMS error } & \multirow[b]{2}{*}{$x$} & \multicolumn{2}{|l|}{ MA error } \\
\hline & $O\left(h_{j}^{2}\right)$ method & $O\left(h_{j}^{3}\right)$ method & & $\bar{O}\left(h^{4}\right)$ method & Wazwaz [20] \\
\hline 8 & $2.3784 \mathrm{e}-05$ & $1.3919 \mathrm{e}-07$ & 0.2 & $5.95 \mathrm{e}-09$ & $1.3 e-08$ \\
\hline 16 & $1.0736 \mathrm{e}-05$ & $3.1300 \mathrm{e}-08$ & 0.4 & $8.06 \mathrm{e}-09$ & $2.3 e-08$ \\
\hline 32 & $6.2320 \mathrm{e}-06$ & $1.2854 \mathrm{e}-08$ & 0.6 & $7.26 \mathrm{e}-09$ & $2.4 \mathrm{e}-08$ \\
\hline 64 & $4.2796 \mathrm{e}-06$ & $8.0985 e-09$ & 0.8 & $4.34 \mathrm{e}-09$ & $1.7 e-08$ \\
\hline
\end{tabular}

Table 4 Example 6.4

\begin{tabular}{rlllll}
\hline $\boldsymbol{N}$ & RMS error & & & MA error & \\
\cline { 2 - 3 } & $\mathbf{O}\left(\boldsymbol{h}_{\boldsymbol{j}}^{\mathbf{2}}\right)$ method & $\mathbf{O}\left(\boldsymbol{h}_{\boldsymbol{j}}^{\mathbf{3}}\right)$ method & & $\mathbf{O}\left(\boldsymbol{h}^{\mathbf{4}}\right)$ method & Arshad et al. [33] \\
\hline 8 & $2.6861 \mathrm{e}-04$ & $2.0392 \mathrm{e}-06$ & $1.9385 \mathrm{e}-07$ & $7.02 \mathrm{e}-06$ \\
16 & $9.2450 \mathrm{e}-05$ & $4.6577 \mathrm{e}-07$ & $1.2204 \mathrm{e}-08$ & $4.35 \mathrm{e}-06$ \\
32 & $5.2686 \mathrm{e}-05$ & $2.2137 \mathrm{e}-07$ & $7.6317 \mathrm{e}-10$ & $7.87 \mathrm{e}-07$ \\
\hline
\end{tabular}


Table 5 Example 6.5

\begin{tabular}{|c|c|c|c|c|c|}
\hline \multirow[t]{2}{*}{$N$} & \multicolumn{2}{|l|}{ RMS error } & \multirow[b]{2}{*}{$x$} & \multicolumn{2}{|l|}{ RE error } \\
\hline & $O\left(h_{j}^{2}\right)$ method & $O\left(h_{j}^{3}\right)$ method & & $\bar{O}\left(h^{4}\right)$ method & Fazhan et al. [34] \\
\hline 40 & $9.6364 \mathrm{e}-02$ & $8.3838 e-03$ & 0.40 & $2.9483 e-05$ & $7.5000 \mathrm{e}-04$ \\
\hline 80 & $6.8221 \mathrm{e}-02$ & $5.9544 \mathrm{e}-03$ & 0.56 & $2.9671 \mathrm{e}-05$ & $7.5000 \mathrm{e}-04$ \\
\hline 160 & $4.8385 \mathrm{e}-02$ & $4.2234 \mathrm{e}-03$ & 0.72 & $2.9898 \mathrm{e}-05$ & $7.2000 \mathrm{e}-04$ \\
\hline
\end{tabular}

Table 6 Example 6.6

\begin{tabular}{|c|c|c|c|c|}
\hline \multirow[t]{2}{*}{$\mathbf{N}$} & \multicolumn{2}{|l|}{ RMS error } & \multicolumn{2}{|l|}{ MA error } \\
\hline & $O\left(h_{j}^{2}\right)$ method & $O\left(h_{j}^{3}\right)$ method & $\boldsymbol{O}\left(h^{4}\right)$ method & $\mathrm{COC}^{*}$ \\
\hline 8 & $9.9887 e-05$ & $1.3841 \mathrm{e}-05$ & $3.0756 e-05$ & - \\
\hline 16 & $6.1365 \mathrm{e}-05$ & $1.7426 \mathrm{e}-06$ & $1.8795 \mathrm{e}-06$ & 4.0324 \\
\hline 32 & $4.3896 e-05$ & $5.1946 \mathrm{e}-07$ & $1.1320 \mathrm{e}-07$ & 4.0534 \\
\hline 64 & $3.1303 e-05$ & $3.1459 e-07$ & $6.8994 \mathrm{e}-09$ & 4.0362 \\
\hline
\end{tabular}

Table 7 Example 6.7

\begin{tabular}{rllll}
\hline $\boldsymbol{N}$ & \multicolumn{2}{l}{ RMS error } & \multicolumn{2}{l}{ MA error } \\
\cline { 2 - 5 } & $\mathbf{O}\left(\boldsymbol{h}_{\boldsymbol{j}}^{\mathbf{2}}\right)$ method & $\mathbf{O}\left(\boldsymbol{h}_{\boldsymbol{j}}^{\mathbf{3}}\right)$ method & O( $\left.\boldsymbol{h}^{\mathbf{4}}\right)$ method & COC $^{*}$ \\
\hline 8 & $3.4729 \mathrm{e}-04$ & $1.0887 \mathrm{e}-05$ & $2.4630 \mathrm{e}-05$ & - \\
16 & $2.3177 \mathrm{e}-05$ & $2.9061 \mathrm{e}-06$ & $1.8929 \mathrm{e}-06$ & 3.7017 \\
32 & $3.8023 \mathrm{e}-05$ & $1.4735 \mathrm{e}-06$ & $1.3820 \mathrm{e}-07$ & 3.7757 \\
64 & $4.1196 \mathrm{e}-05$ & $1.2815 \mathrm{e}-06$ & $9.9099 \mathrm{e}-09$ & 3.8018 \\
\hline
\end{tabular}

Table 8 Example 6.8

\begin{tabular}{|c|c|c|c|c|}
\hline \multirow[t]{2}{*}{$\mathbf{N}$} & \multicolumn{2}{|l|}{ RMS error } & \multicolumn{2}{|l|}{ MA error } \\
\hline & $\bar{O}\left(h_{j}^{2}\right)$ method & $O\left(h_{j}^{3}\right)$ method & $\bar{O}\left(h^{4}\right)$ method & $\mathrm{COC}^{*}$ \\
\hline 8 & $2.8679 \mathrm{e}-03$ & $1.8940 \mathrm{e}-05$ & $2.5391 \mathrm{e}-06$ & - \\
\hline 16 & $1.1316 \mathrm{e}-03$ & $3.3587 e-06$ & $1.6213 e-07$ & 3.9691 \\
\hline 32 & $7.0535 \mathrm{e}-04$ & $1.3791 \mathrm{e}-06$ & $1.0187 \mathrm{e}-08$ & 3.9923 \\
\hline 64 & $6.4066 \mathrm{e}-04$ & $1.1509 \mathrm{e}-06$ & $6.3748 \mathrm{e}-10$ & 3.9982 \\
\hline
\end{tabular}

Example 6.1 Consider the fourth-order linear boundary value problem of the form [31]

$$
\begin{aligned}
& \frac{d^{4} y}{d x^{4}}(x)-y(x)=-8 x \cos (x)-12 \sin (x), \quad 0 \leq x \leq 1, \\
& y(0)=y(1)=0, \quad \frac{d^{2}}{d x^{2}} y(0)=0, \quad \frac{d^{2}}{d x^{2}} y(1)=2 \sin (1)+4 \cos (1) .
\end{aligned}
$$

The exact solution is given by $y(x)=\left(x^{2}-1\right) \sin (x)$. The RMS errors for a fixed value $\sigma=0.9$ and MA error for $\sigma=1$ are tabulated in Table 1 . The graph of the exact solution versus the approximate solution using the fourth-order method for $N=32$ is given by Figure 1.

Example 6.2 We consider the sixth-order linear boundary value problem [21, 32]

$$
\begin{aligned}
& \left(\frac{d^{6}}{d x^{6}}+1\right) y(x)=6(2 x \cos (x)+5 \sin (x)), \quad 0 \leq x \leq 1 \\
& y(0)=y(1)=0, \quad \frac{d^{2}}{d x^{2}} y(0)=0, \quad \frac{d^{2}}{d x^{2}} y(1)=2 \sin (1)+4 \cos (1),
\end{aligned}
$$




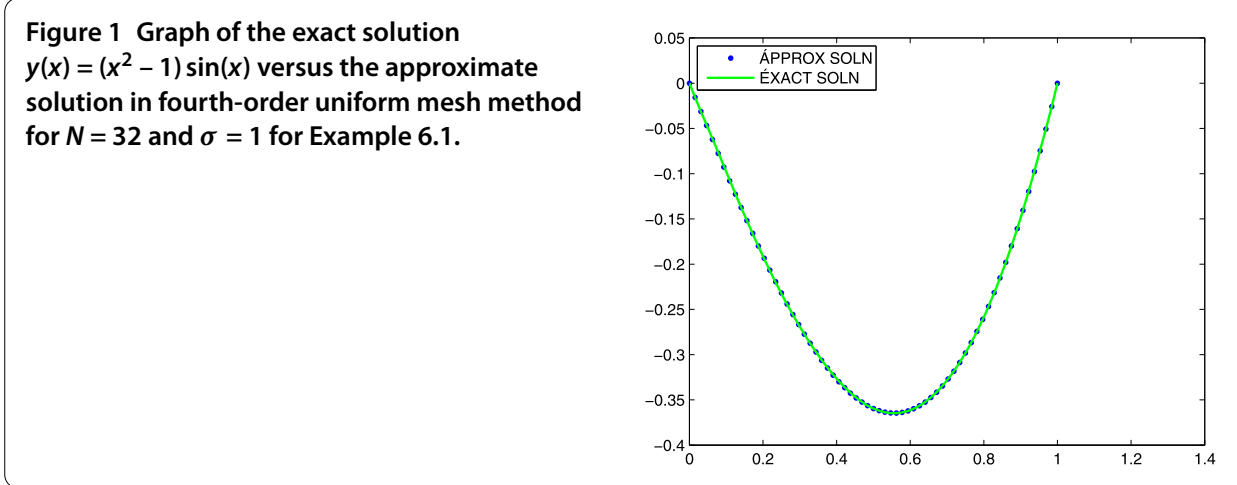

Figure 2 Graph of the exact solution $y(x)=\left(x^{2}-1\right) \sin (x)$ versus the approximate solution in fourth-order uniform mesh method for $N=32$ and $\sigma=1$ for Example 6.2.

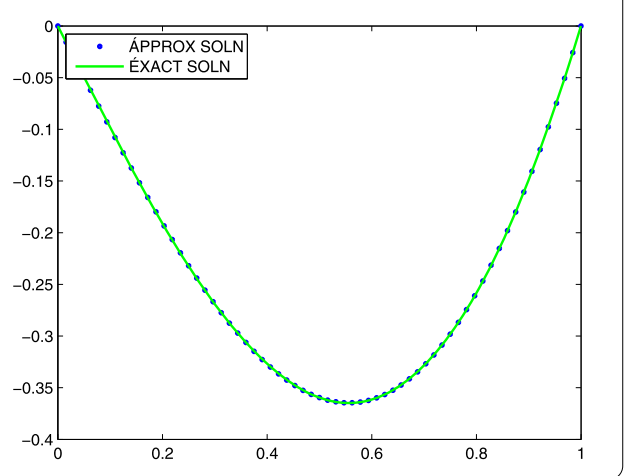

$$
\frac{d^{4}}{d x^{4}} y(0)=0, \quad \frac{d^{4}}{d x^{4}} y(1)=-12 \sin (1)-8 \cos (1) .
$$

The exact solution is given by $y(x)=\left(x^{2}-1\right) \sin (x)$. The RMS errors for a fixed value $\sigma=0.9$ and MA error for $\sigma=1$ are tabulated in Table 2. The graph of the exact solution versus the approximate solution using the fourth-order method for $N=32$ is given by Figure 2 .

Example 6.3 Consider the fourth-order non-linear boundary value problem [20]:

$$
\begin{aligned}
& \frac{d^{4} y}{d x^{4}}(x)=-6 \exp (-4 y(x)), \quad 0 \leq x \leq 4-e, \\
& y(0)=1, \quad y(4-e)=\ln (4), \quad \frac{d^{2}}{d x^{2}} y(0)=-\frac{1}{e^{2}}, \quad \frac{d^{2}}{d x^{2}} y(4-e)=-\frac{1}{16} .
\end{aligned}
$$

The exact solution is given by $y(x)=\ln (e+x)$. The RMS errors for a fixed value $\sigma=0.9$ and MA error for $\sigma=1$ are tabulated in Table 3. The graph of the exact solution versus the approximate solution using the fourth-order method for $N=64$ is given by Figure 3 .

Example 6.4 Consider the sixth-order non-linear boundary value problem [33]:

$$
\begin{aligned}
& \frac{d^{6} y}{d x^{6}}=\exp (-x) y^{2}, \quad 0 \leq x \leq 1, \\
& y(0)=\frac{d^{2}}{d x^{2}} y(0)=\frac{d^{4}}{d x^{4}} y(0)=1, \quad y(1)=\frac{d^{2}}{d x^{2}} y(1)=\frac{d^{4}}{d x^{4}} y(1)=e .
\end{aligned}
$$




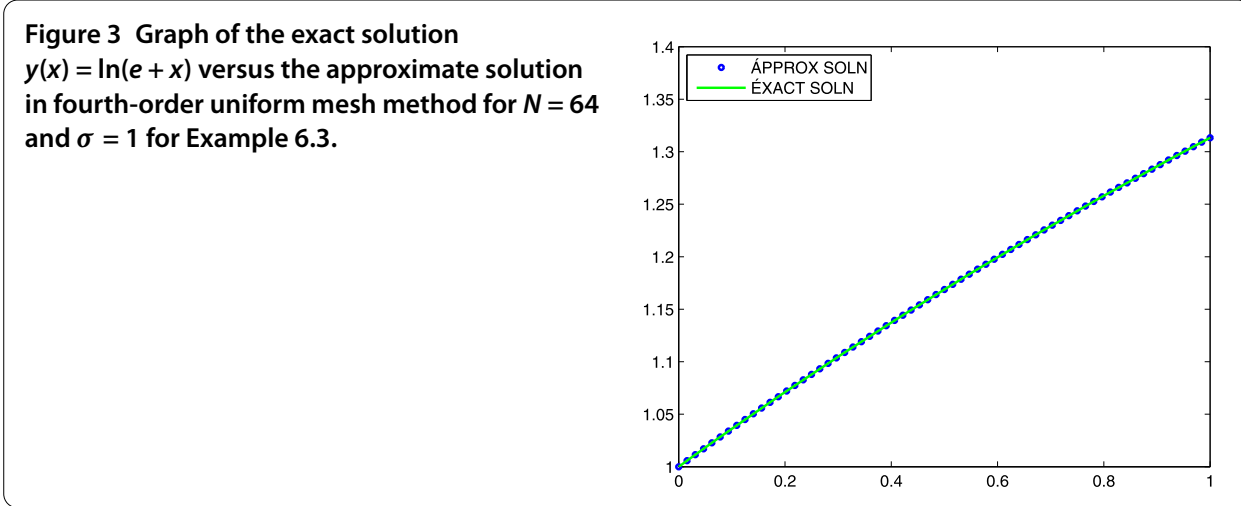

Figure 4 Graph of the exact solution $y(x)=\exp (x)$ versus the approximate solution in fourth-order uniform mesh method for $N=32$ and $\sigma=1$ for Example 6.4.

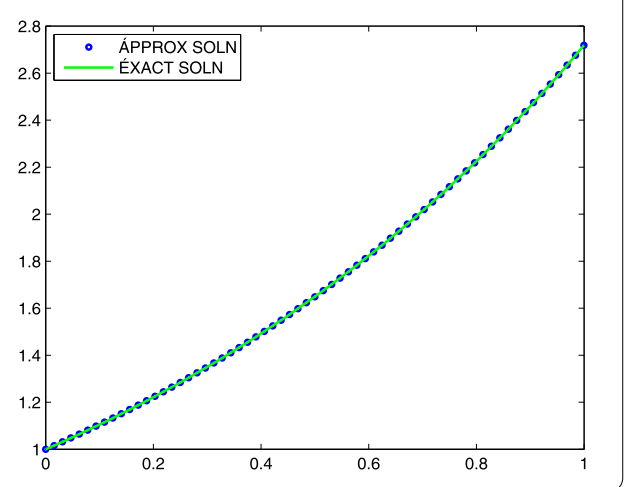

The exact solution is given by $y(x)=\exp (x)$. The RMS errors for a fixed value $\sigma=0.9$ and MA error for $\sigma=1$ are tabulated in Table 4 . The graph of the exact solution versus the approximate solution using the fourth-order method for $N=32$ is given by Figure 4 .

Example 6.5 Consider a sixth-order singular boundary value problem of the form [34]:

$$
\begin{aligned}
& 15 x(1-x) \frac{d^{6} y}{d x^{6}}+x \frac{d^{5} y}{d x^{5}}+(25+\exp (2 x)) \frac{d^{4} y}{d x^{4}}+(5+\exp (x)) \frac{d^{2} y}{d x^{2}}+x y=f(x), \quad 0<x<1, \\
& y(0)=y(1)=0, \quad \frac{d^{2}}{d x^{2}} y(0)=\frac{d^{2}}{d x^{2}} y(1)=0, \quad \frac{d^{4}}{d x^{4}} y(0)=\frac{d^{4}}{d x^{4}} y(1)=0,
\end{aligned}
$$

where

$$
f(x)=\pi^{2} x \cos (\pi x)+\left(-(5+\exp (x)) \pi^{2}+(25+\exp (2 x)) \pi^{4}+x+15 \pi^{6}(x-1) x\right) \sin (\pi x) .
$$

The exact solution is given by $y(x)=\sin (\pi x)$. The RMS errors for a fixed value $\sigma=0.9$ and RE error for $\sigma=1$ are tabulated in Table 5. The graph of the exact solution versus the approximate solution using the fourth-order method for $N=160$ is given by Figure 5 .

Example 6.6 Consider a fourth-order non-linear singular boundary value problem of the form

$$
\left(\frac{d^{4}}{d x^{4}}+\frac{4}{x} \frac{d^{3}}{d x^{3}}\right) y=y^{2}+\frac{\cos (x)}{x}(x \sin (2 x)-4), \quad 0<x \leq 1 .
$$


Figure 5 Graph of the exact solution $y(x)=\sin (\pi x)$ versus the approximate solution in fourth-order uniform mesh method for $N=160$ and $\sigma=1$ for Example 6.5.

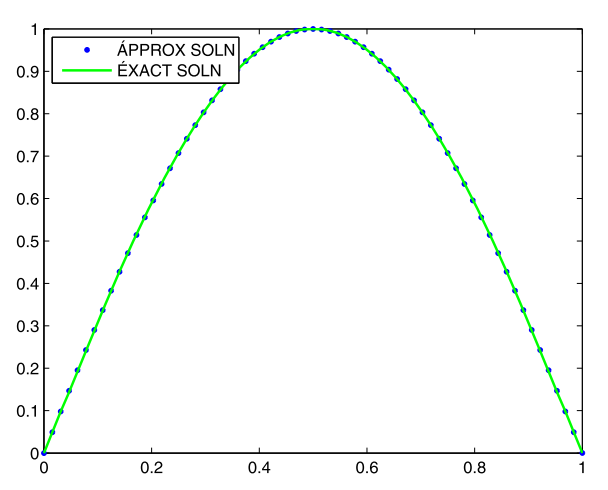

Figure 6 Graph of the exact solution $y(x)=\sin (x)$ versus the approximate solution in fourth-order uniform mesh method for $N=64$ and $\sigma=1$ for Example 6.6.

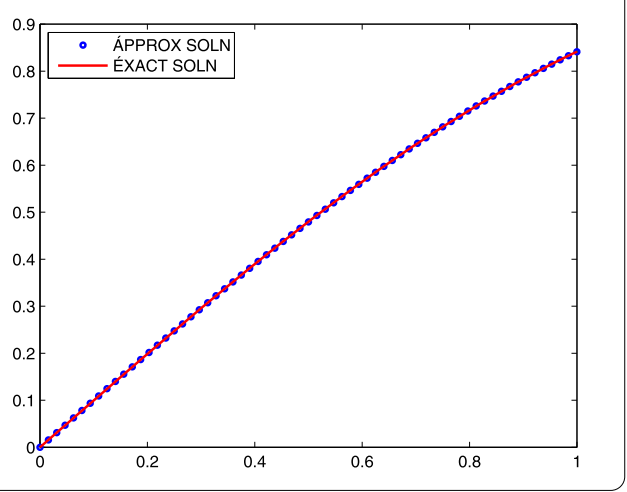

The exact solution is given by $y(x)=\sin (x)$. The boundary conditions are obtained from the exact solution by a test procedure. The RMS errors for a fixed value $\sigma=0.9$ and MA error for $\sigma=1$ are tabulated in Table 6 . The graph of the exact solution versus the approximate solution using the fourth-order method for $N=64$ is given by Figure 6 .

Example 6.7 We consider a sixth-order non-linear singular boundary value problem of the form

$$
\left(\frac{d^{6}}{d x^{6}}+\frac{6}{x} \frac{d^{5}}{d x^{5}}+2\right) y=e^{y}+6 e^{x}\left(\frac{1+x}{x}\right), \quad 0<x \leq 1
$$

The exact solution is given by $y(x)=\exp (x)$. The boundary conditions are obtained from the exact solution by a test procedure. The RMS errors for a fixed value $\sigma=0.9$ and MA error for $\sigma=1$ are tabulated in Table 7. The graph of the exact solution versus the approximate solution using the fourth-order method for $N=64$ is given by Figure 7 .

Example 6.8 We consider a system of second-order boundary value problem of the form:

$$
\begin{aligned}
& \frac{d^{2} y}{d x^{2}}=y \frac{d z}{d x}+z \frac{d y}{d x}+f(x), \quad 0 \leq x \leq 1, \\
& \frac{d^{2} z}{d x^{2}}=z \frac{d z}{d x}+y \frac{d y}{d x}+g(x) .
\end{aligned}
$$

The exact solution is given by $y(x)=\sinh (x), z(x)=\cosh (x)$. The boundary conditions are obtained from the exact solution by a test procedure. The RMS errors for a fixed value 


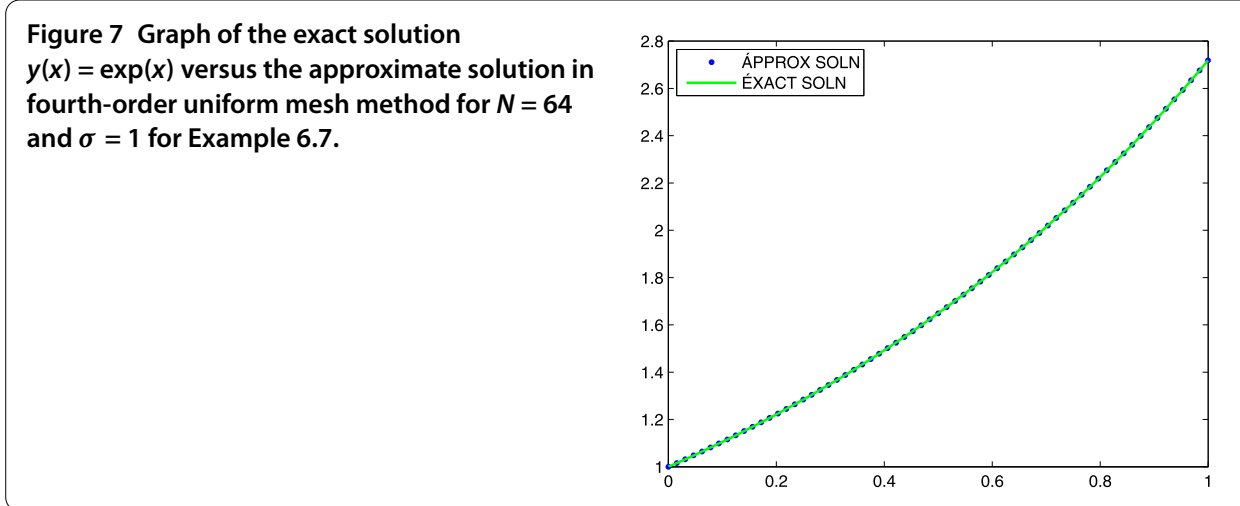

Figure 8 Graph of the exact solution $y(x)=\sinh (x)$ versus the approximate solution in fourth-order uniform mesh method for $N=64$ and $\sigma=1$ for Example 6.8.

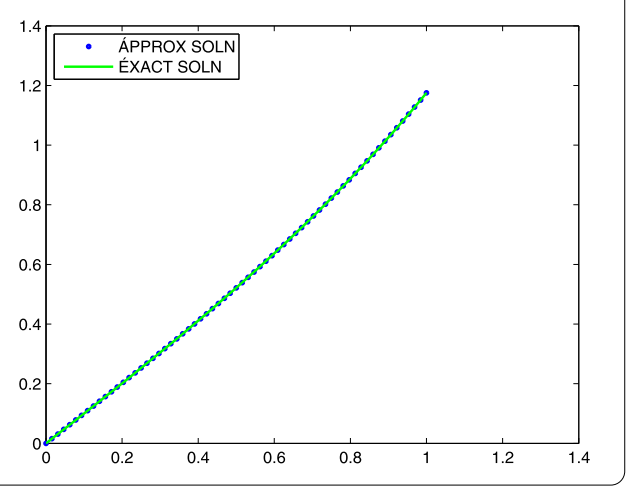

$\sigma=0.9$ and MA error for $\sigma=1$ are tabulated in Table 8. The graph of the exact solution versus the approximate solution using the fourth-order method for $N=64$ is given by Figure 8.

\section{Conclusion}

We derived second- as well as third-order variable-mesh schemes for solving linear, nonlinear even-order cases and systems of second-order boundary value problems. Although, in this paper, only fourth-order and sixth-order non-linear and linear singular boundary value problems are considered, the method is general enough to implement in the case of higher even-order linear and non-linear singular boundary value problems.

Tables 1-4 shows the presence of refinement in results when compared with other linear and non-linear boundary value problems solved by computational methods using the quintic non-polynomial spline, a septic non-polynomial and a parametric spline, a sextic spline, and a modified Adomian decomposition method. In Table 5 results have been compared with solutions derived analytically by reproducing kernel method [34]. Computationally our methods seem to be more viable due to the usage of only three grid points at a time which leads to solving of a tri-diagonal matrix. However, in the case of the quintic, the sextic and septic splines a higher number of grid points are required, which results in pentadiagonal or septadiagonal matrices. Also, in the end we have solved fourthand sixth-order non-linear singular and a system of second-order boundary value problems. As per the literature available, such a class of boundary value problems has not been solved so far. Therefore, due to the unavailability of any prior results we were unable to 
present a comparative study. Hence we have compared our own results in Table 6, 7, 8 and have also provided the computational order of convergence $\left(\mathrm{COC}^{*}\right)$ for the uniform mesh method. Our methods are also applicable to problems in cartesian as well as polar coordinates with minor modifications and even higher-order singularly perturbed boundary value problems can be solved easily due to the use of a variable mesh.

\section{Acknowledgements}

The authors thank the anonymous reviewers for their valuable comments, which substantially improved the paper.

\section{Competing interests}

The authors declare that they have no competing interests.

Authors' contributions

All authors drafted the manuscript, and they read and approved the final version.

\section{Author details}

'Department of Mathematics, South Asian University, New Delhi, 110021, India. ²Department of Mathematics, Lady Shri Ram College for Women, University of Delhi, New Delhi, 110024, India. ${ }^{3}$ Department of Mathematics, Jamia Millia Islamia, New Delhi, 110025, India.

\section{Publisher's Note}

Springer Nature remains neutral with regard to jurisdictional claims in published maps and institutional affiliations.

Received: 19 July 2017 Accepted: 27 September 2017 Published online: 13 October 2017

\section{References}

1. Keller, HB: Numerical Methods for Two Point Boundary Value Problems. Blaisdell Publications Co., New York (1968)

2. Bernis, F: Compactness of the support in convex and non-convex fourth order elasticity problem. Nonlinear Anal. 6 , 1221-1243 (1982)

3. Glatzmaier, GA: Numerical simulations of stellar convection dynamics at the base of the convection zone. Geophys. Astrophys. Fluid Dyn. 31, 137-150 (1985)

4. Toomre, J, Zahn, JR, Latour, J, Spiegel, EA: Stellar convection theory II: single-mode study of the second convection zone in A-type stars. Astrophys. J. 207, 545-563 (1976)

5. Terril, RM: Laminar flow in a uniformly porous channel. Aeronaut. Q. 15(3), 299-310 (1964)

6. Chandrasekhar, S: Hydrodynamic and Hydromagnetic Stability. Clarendon Press, Oxford (1961). Reprinted: Dover Books, New York, 1981

7. Aftabizadeh, AR: Existence and uniqueness theorems for fourth-order boundary value problems. J. Math. Anal. Appl. $116(2), 415-426(1986)$

8. Zill, DG, Cullen, MR: Differential Equations with Boundary-Value Problems, 5th edn. Brooks/Cole, Pacific Grove (2001)

9. O'Regan, D: Solvability of some fourth (and higher) order singular boundary value problems. J. Math. Anal. Appl. 161(1), 78-116 (1991)

10. Agarwal, RP: Boundary value problems for higher order differential equations. Bull. Inst. Math. Acad. Sin. 9(1), 47-61 (1981)

11. Mohanty, RK, Evans, DJ: A fourth order accurate cubic spline alternating group explicit method for non-linear singular two point boundary value problems. Int. J. Comput. Math. 80, 479-492 (2003)

12. Mohanty, RK, Sachdev, PL, Jha, N: An $O\left(h^{4}\right)$ accurate cubic spline TAGE method for non-linear singular two point boundary value problems. Appl. Math. Comput. 158, 853-868 (2004). doi:10.1016/j.amc.2003.08.145

13. Mohanty, RK, Khosla, N: A third order accurate variable mesh TAGE iterative method for the numerical solution of two point non-linear singular boundary value problems. Int. J. Comput. Math. 82, 1261-1273 (2005)

14. Kadalbajoo, MK, Bawa, RK: Third order variable-mesh cubic spline methods for singularly perturbed boundary value problems. Appl. Math. Comput. 59, 117-129 (1993)

15. Mohanty, RK, Jha, N, Evans, DJ: Spline in compression method for the numerical solution of singularly perturbed two point singular boundary value problems. Int. J. Comput. Math. 81, 615-627 (2004)

16. Mohanty, RK, Jha, N: A class of variable mesh spline in compression methods for singularly perturbed two point singular boundary value problems. Appl. Math. Comput. 168, 704-716 (2005). doi:10.1016/j.amc.2004.09.049

17. Mohanty, RK, Evans, DJ, Arora, U: Convergent spline in tension methods for singularly perturbed two point singular boundary value problems. Int. J. Comput. Math. 82, 55-66 (2005)

18. Mohanty, RK, Arora, U: A family of non-uniform mesh tension spline methods for singularly perturbed two point singular boundary value problems with significant first derivatives. Appl. Math. Comput. 172, 531-544 (2006)

19. Mohanty, RK, Evans, DJ, Khosla, $\mathrm{N}$ : $\mathrm{An} \mathrm{O}\left(h^{3}\right)$ non-uniform mesh cubic spline TAGE method for non-linear singular two-point boundary value problems. Int. J. Comput. Math. 82, 1125-1139 (2005)

20. Wazwaz, A-M: The numerical solution of special fourth-order boundary value problem by the modified decomposition method. Int. J. Comput. Math. 79(3), 345-356 (2002)

21. Akram, G, Siddiqi, SS: Solution of sixth order boundary value problems using non-polynomial spline technique. Appl. Math. Comput. 181(1), 708-720 (2006)

22. Talwar, J, Mohanty, RK: A class of numerical methods for the solution of fourth-order ordinary differential equations in polar coordinates. Adv. Numer. Anal. 2012, Article ID 626419 (2012) 
23. Twizell, EH, Boutayeb, A: Finite-difference methods for the solution of special eighth-order boundary value problems. Int. J. Comput. Math. 48, 63-75 (1993)

24. Akram, G, Ur Rehman, H: Numerical solution of eighth order boundary value problems in reproducing kernel space. Numer. Algorithms 62, 527-540 (2013)

25. Siddiqi, SS, Akram, G: Solution of eighth-order boundary value problems using the non-polynomial spline technique. Int. J. Comput. Math. 84(3), 347-368 (2007)

26. Siddiqi, SS, Akram, G: Septic spline solutions of sixth-order boundary value problems. J. Comput. Appl. Math. 215, 288-301 (2008)

27. Liu, GR, Wu, TY: Differential quadrature solutions of eighth order boundary value differential equations. J. Comput. Appl. Math. 145, 223-235 (2002)

28. Jha, N, Kumar, N: A fourth-order accurate quasi-variable mesh compact finite-difference scheme for two-space dimensional convection-diffusion problems. Adv. Differ. Equ. 2017, Article ID 64 (2017). doi:10.1186/s13662-017-1115-4

29. Jain, MK: Numerical Solution of Differential Equations, 3rd edn. New Age International, New Delhi (2014)

30. Varga, RS: Matrix Iterative Analysis. Prentice-Hall International, Englewood Cliffs (1962)

31. Ramadan, MA, Lashien, IF, Zahra, WK: Quintic nonpolynomial spline solutions for fourth order two-point boundary value problem. Commun. Nonlinear Sci. Numer. Simul. 14, 1105-1114 (2009)

32. Siddiqi, SS, Twizell, EH: Spline solutions of linear sixth-order boundary value problems. Int. J. Comput. Math. 60, 295-304 (1996)

33. Khan, A, Khandelwal, P: Solution of non-linear sixth-order two point boundary-value problems using parametric septic splines. Int. J. Nonlinear Sci. 12(2), 184-195 (2011)

34. Geng, F, Ye, Y: Solving analytically singular sixth-order boundary value problems. Int. J. Math. Anal. 3(39), 1945-1952 (2009)

\section{Submit your manuscript to a SpringerOpen ${ }^{\circ}$ journal and benefit from:}

- Convenient online submission

- Rigorous peer review

Open access: articles freely available online

- High visibility within the field

- Retaining the copyright to your article

Submit your next manuscript at springeropen.com 